\title{
Optimization of energy extraction in transverse galloping
}

\author{
F. Sorribes-Palmer*, A. Sanz-Andres
}

\begin{abstract}
A B S T R A C T
A numerical method to analyse the stability of transverse galloping based on experimental measurements, as an alternative method to polynomial fitting of the transverse force coefficient $C_{z}$, is proposed in this paper. The Glauert-Den Hartog criterion is used to determine the region of angles of attack (pitch angles) prone to present galloping. An analytic solution (based on a polynomial curve of $C_{z}$ ) i s u sedtovalid a t e the method a nd t o evaluate the discretization errors. Several bodies (of biconvex, Dshape and rhomboidal cross sections) have been tested in a wind tunnel and the stability of the galloping region has been analysed with the new method. An algorithm to determine the pitch angle of the body that allows the maximum value of the kinetic energy of the flow to be extracted is presented.
\end{abstract}

\section{Introduction}

Aeroelastic phenomena are still an important issue in the design of engineering structures (aircraft, wind turbines, bridges, tall buildings, power lines, etc.), among them we find transverse galloping, wake galloping, flutter, vortex shedding, torsional divergence and buffeting. This paper focuses on the transverse galloping, whose theoretical foundations can be found in Crawley et al. (1995). The galloping occurs when the aerodynamic forces associated with the motion of the structure have a destabilizing character Barrero-Gil (2008). The galloping is caused by a coupling between the aerodynamic forces and the across-wind oscillations induced in the structure, which change the angle of attack, which in turn vary the aerodynamic forces modifying the dynamical response of the structure. The structure, which usually has low stiffness and low damping, moves in the direction normal to the average wind speed. This motion is characterized by oscillations of large amplitude and low frequency. The most important parameters which influence galloping (considered here as a one-degree-of-freedom oscillator subjected to aerodynamic forces) are the geometric shape, the angle of incidence, the speed of flow, the density and viscosity of the fluid, the turbulence intensity of the flow, and the system's mechanical properties (mass, stiffness and damping).

Until now, the tool usually used to analyse the stability in transverse galloping of a body is based on the shape of the curve of the dimensionless coefficient of the aerodynamic force transverse to the incident flow $C_{z}(\alpha), \alpha$ being the angle of attack, and its expansion in powers of $\alpha$ or $\tan \alpha$ (Alonso et al., 2009). For the study of a particular case this method can be complex and tedious, because it requires a polynomial fitting to the experimental results. This rises concerns about the suitable number of points to consider, and the range of amplitude of motion around the pitch angle of the body to be taken into consideration in the polynomial fitting. Note that if the amplitude of the oscillations exceeds the range of validity of the fitting, the dynamics obtained from the analysis will differ from its real behaviour. For the analysis of a generic configuration, the approach in Barrero-Gil et al. (2009), which concludes that the number of inflection points in the curve 


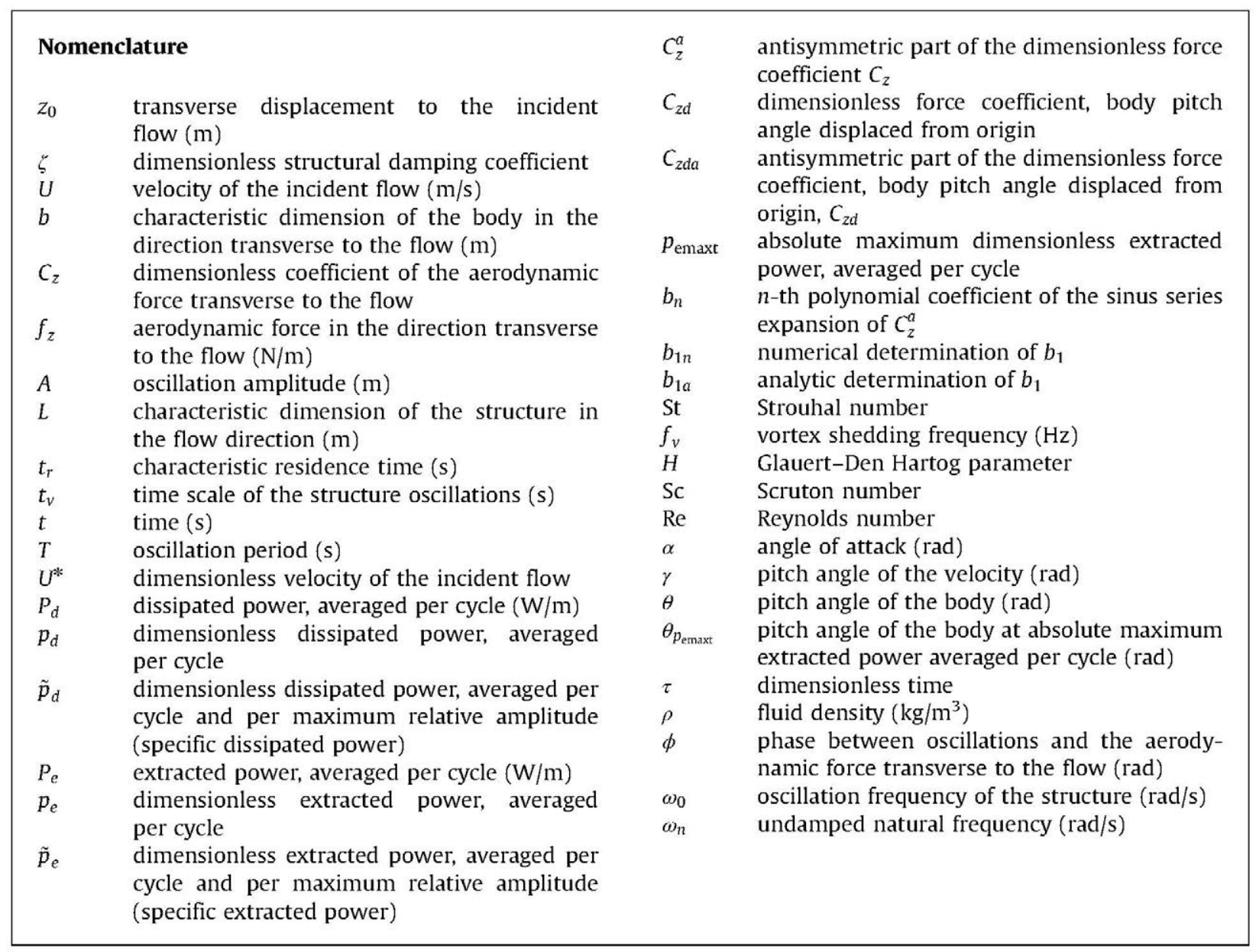

$C_{z}(\alpha)$ determines the regions where hysteresis in galloping may appear, is restricted to the simple cases that allow a low order polynomial fitting, with limited physical meaning.

In Ng et al. (2005), high order polynomials (ninth and eleventh) are used to fit the curve of $C_{z}(\alpha)$ in order to reveal more flow physics. But no additional positive real roots appear, only extra negative real and/or complex roots are obtained, concluding that a seventh order polynomial is sufficient to capture the hysteresis phenomenon in a squared cylinder.

In Abdelkefi et al. (2013) the polynomial fitting is also used to approximate the aerodynamic force to model a galloping-based piezoaeroelastic energy harvester. A linear and a nonlinear analysis are performed to determine the effects of the electrical load resistance and the cross-section geometry on the onset of galloping, which is due to a Hopf bifurcation. The results show that the maximum levels of harvested power are accompanied with minimum transverse displacement amplitudes.

The aim of this paper is to present a new method that does not require the power series expansion for the abovementioned fitting, but is based on the use of the curve $C_{z}(\alpha)$ obtained directly from the experimental data, without requiring any polynomial fitting. Depending on the shape of the curve $C_{z}(\alpha)$ a hysteresis phenomenon may appear linked to the separation and re-adherence of the boundary layer on the upper or lower surfaces of the body. In this case, different amplitudes of the limit cycle oscillations can be achieved depending on whether the flow speed is increasing or decreasing.

In this paper an alternative method to the polynomial fitting analysis is proposed to study the stability of the phenomenon of transverse galloping.

This paper is organized as follows: the mathematical model that describes the transverse galloping and the method to analyse the stability is presented in Section 2. The experimental set-up used in the measurements is described in Section 3. In Section 4 the results obtained from the application of the method to experimental measurements are shown. Finally, in Section 5, conclusions are drawn.

\section{Mathematical model}

Since the dynamical behaviour of a structure placed inside a fluid flow can be a very complex problem, it has become customary in the study of transverse galloping to analyse the problem using a simplified model consisting of a linear one-degree-of-freedom 


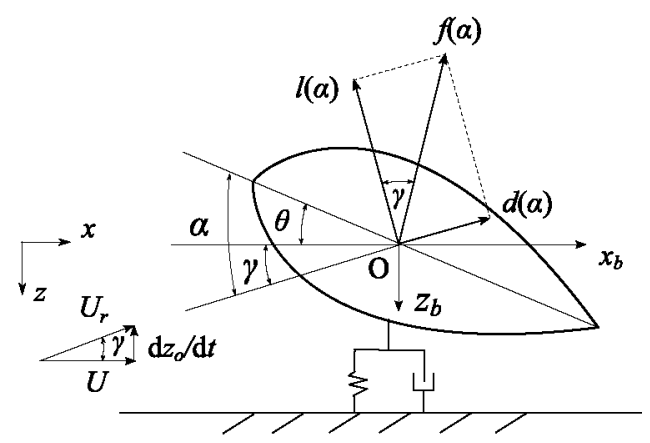

Fig. 1. Schematic representation of aerodynamic forces and the angle of attack, $\alpha$, pitch angle of the velocity, $\gamma$, pitch angle of the body, $\theta ; z, x$ are the inertial reference frame and $z_{b}, x_{b}$ are the reference frame attached to the body.

oscillator, a structure which is slender enough to consider two-dimensional flow, and neglecting the effect of the incident flow turbulence (Parkinson and Smith, 1964). The simplified model of the system is shown in Fig. 1.

Under these conditions, common in fluid-structure interaction analysis, the equation that describes the dynamic of transverse galloping can be expressed as

$$
m\left(\ddot{z}_{0}+2 \zeta \omega_{n} \dot{z}_{0}+\omega_{n}^{2} z_{0}\right)=f_{z}(\alpha)=\frac{1}{2} \rho U^{2} b C_{z}(\alpha),
$$

where $z_{0}$ denotes the displacement of the point $O$ transverse to the incident flow, $\omega_{n}$ is the undamped natural frequency, $\zeta$ the dimensionless structural damping coefficient, $U$ the velocity of the incident flow, $\rho$ the fluid density, $b$ the characteristic dimension of the body in the direction transverse to the flow, $f_{z}(\alpha)$ the aerodynamic force in the direction transverse to the flow, and $C_{z}(\alpha)$ the dimensionless coefficient of the aerodynamic force transverse to the flow

$$
C_{z}(\alpha)=-\frac{c_{l}(\alpha)+c_{d}(\alpha) \tan \alpha}{\cos \alpha}
$$

where

$$
\tan \alpha=\frac{1}{U} \frac{\mathrm{d} z_{0}}{\mathrm{~d} t}
$$

This expression refers to the study of the oscillatory motion around a pitch angle of the body $\theta$ considered as reference, with $\theta=0$ as origin. If the lift $c_{l}(\theta)$ and $\operatorname{drag} c_{d}(\theta)$ coefficients determined in static conditions $(\theta=\alpha)$ are defined as a function of the pitch angle of the body $\theta$, and the reference point of study is $\theta_{0} \neq 0$, doing the change of variable $\alpha=\theta-\theta_{0}$, the functions $c_{l}(\alpha)$ and $c_{d}(\alpha)$ can be generated for each $\theta_{0}$.

The hypothesis of quasi-steady aerodynamic behaviour can be assumed, since the transverse galloping appears at high enough velocities, such that the characteristic residence time scale of the flow $\left(t_{r} \sim L / U\right)$ is small compared with the time scale of the change in boundary conditions of the flow, which can be evaluated as the period of the structure oscillations $\left(t_{v} \sim 1 / \omega_{0}\right)$. In the above expressions $L$ is the characteristic dimension of the structure in the direction of the flow and $\omega_{0}$ the oscillation frequency of the structure.

The vortex shedding phenomenon is not taken into account here because, at the flow spees considered, the vortex shedding frequency $f_{v}$ is much higher than the oscillation frequency of the body $\omega_{0} / 2 \pi$ and both the problems can be decoupled. Therefore, the transverse galloping phenomenon can be analysed using static measurements in a wind tunnel at a pitch angle coincident with the instantaneous angle of the incident flow.

The solution to the motion equation (1) by the Krylov-Bogoliubov method is based on assuming that the system is an undamped oscillator slightly perturbed by both a small damping $\zeta$, and an aerodynamic force transverse to the flow $f_{z}(\alpha)$, so that the motion of the body can be considered harmonic

$$
z_{0}(t)=A \cos \left(\omega_{n} t+\phi\right),
$$

where the amplitude, $A$, and the phase, $\phi$, are slowly varying functions of time, $t$. The dimensionless dissipated power averaged per cycle (due to friction, or another similar mechanism) can be defined as

$$
p_{d}=\frac{P_{d}}{\frac{1}{2} \rho U^{3} b}
$$

where $P_{d}$ is the dissipated power averaged per cycle,

$$
P_{d}=\frac{1}{T} \int_{0}^{T} 2 m \zeta \omega_{n}\left(\frac{\mathrm{d} z_{0}}{\mathrm{~d} t}\right)^{2} \mathrm{~d} t=\frac{1}{2 \pi} \int_{0}^{2 \pi} 2 m \zeta \omega_{n}^{3} A^{2} \sin ^{2} \tau \mathrm{d} \tau,
$$

where $\tau=\omega_{n} t+\phi$ is the dimensionless time, and $T=2 \pi / \omega_{n}$ the oscillation period.

Dividing the amplitude of the oscillations by the characteristic dimension of the body in the direction transverse to the flow $b, z_{0}=b \psi, \psi=(A / b) \cos \tau=A^{*} \cos \tau$, the mass of the body divided by the characteristic mass of the surrounding fluid, 
$m^{*}=m /\left(\rho b^{2}\right)$; and considering the dimensionless velocity $U^{*}=U /\left(\omega_{n} b\right)$, the expression for the transverse velocity of the body obtained is

$$
\frac{\mathrm{d} z_{0}}{\mathrm{~d} t}=-b A^{*} \omega_{n} \sin \tau
$$

The dimensionless dissipated power averaged per cycle defined in (5), using (6) and (7) becomes

$$
p_{d}=\frac{1}{\frac{1}{2} \rho U^{3} b} \frac{b^{2} 2 m \zeta \omega_{n}^{3} A^{* 2}}{2 \pi} \int_{0}^{2 \pi} \sin ^{2} \tau \mathrm{d} \tau=\frac{2 m^{*} \zeta}{U^{*}}\left(\frac{A^{*}}{U^{*}}\right)^{2} .
$$

The extracted power averaged per cycle is

$$
P_{e}=\frac{1}{T} \int_{0}^{T} f_{z} \frac{\mathrm{d} z_{0}}{\mathrm{~d} t} \mathrm{~d} t=\frac{\rho U^{2} b}{2 T} \int_{0}^{T} C_{z} \frac{\mathrm{d} z_{0}}{\mathrm{~d} t} \mathrm{~d} t,
$$

and the dimensionless extracted power averaged per cycle is given by

$$
p_{e}=\frac{P_{e}}{\frac{1}{2} \rho U^{3} b}=\frac{1}{U T} \int_{0}^{T} C_{z} \frac{\mathrm{d} z_{0}}{\mathrm{~d} t} \mathrm{~d} t .
$$

The first step in our study is to prove that the dimensionless extracted power per cycle depends only on the parameter $A^{*} / U^{*}$. To do this, we will consider that the dimensionless coefficient of the aerodynamic force transverse to the flow can always be expanded in power series of $\tan \alpha$

$$
C_{z}(\alpha)=\sum a_{n} \tan ^{n} \alpha,
$$

so the expression of the dimensionless extracted power averaged per cycle (10) becomes

$$
\begin{aligned}
p_{e} & =\frac{1}{U T} \int_{0}^{T}\left(\sum a_{n} \tan ^{n} \alpha\right) \frac{\mathrm{d} z_{0}}{\mathrm{~d} t} \mathrm{~d} t=\frac{b A^{*} \omega_{n}}{U 2 \pi} \int_{0}^{2 \pi} \sum\left(-a_{n} \sin \tau\right)\left(-\frac{b A^{*} \omega_{n} \sin \tau}{U}\right)^{n} \mathrm{~d} \tau \\
& =\frac{1}{2 \pi} \sum a_{n}\left(-\frac{A^{*}}{U^{*}}\right)^{n+1} \int_{0}^{2 \pi}(\sin \tau)^{n+1} \mathrm{~d} \tau=\frac{1}{2}\left[a_{1}\left(\frac{A^{*}}{U^{*}}\right)^{2}+\frac{3}{4} a_{3}\left(\frac{A^{*}}{U^{*}}\right)^{4}+\cdots\right] .
\end{aligned}
$$

Note that in Eq. (12) just the odd terms $n=2 p-1$ contribute, and it can also be concluded that $p_{e}$ is a function depending only on $A^{*} / U^{*}$, making it easier to find the maximum of the dimensionless extracted power per cycle, as this only depends on a single parameter

$$
\left.p_{e}=\left(\frac{A^{*}}{U^{*}}\right)^{2} f\left(\frac{A^{*}}{U^{*}}\right)^{2}\right)
$$

The maximum extracted power, $p_{e \max }$, is obtained for the value of $A^{*} / U^{*}=\left(A^{*} / U^{*}\right)_{p_{\operatorname{emax}}}$ that maximizes $p_{e}$. In equilibrium, the extracted power (13) and the dissipated power (8) are equal, $p_{d}=p_{e}$, from this equation the expression obtained is

$$
\left.\frac{2 m^{*} \zeta}{U^{*}}=f\left(\frac{A^{*}}{U^{*}}\right)^{2}\right)
$$

or its inverse

$$
\left(\frac{A^{*}}{U^{*}}\right)^{2}=g\left(\frac{2 m^{*} \zeta}{U^{*}}\right)
$$

The value $\left(m^{*} \zeta / U^{*}\right)_{p_{\max }}$ determines the combination of parameters that produces the maximum energy extracted. It can be obtained from (14) or (15) once $\left(A^{*} / U^{*}\right)_{p_{\operatorname{emax}}}$ is known from (13). This procedure can also be used even if the coefficients $a_{n}$ of the expansion (11) are unknown, but the function $f\left(A^{*} / U^{*}\right)$ can be determined by other methods (e.g. experimentally).

\subsection{Extracted power in a general case}

In this section we consider a general case, in which $C_{z}(\alpha)$ has a complex shape and cannot be easily fitted to such a low degree polynomial that an appropriate analytic solution can be obtained. We will show that it is possible to calculate the power extracted directly from the experimental data. Let us consider just the antisymmetric part, $C_{z}^{a}(\alpha)$, of the dimensionless coefficient of the aerodynamic force transverse to the flow. Expressions (9) and (10) can be rewritten as

$$
\begin{aligned}
& P_{e}=-\frac{\rho U^{2} b^{2} A^{*}}{2 T} \int_{0}^{T} C_{z}^{a}(\alpha) \sin \tau \mathrm{d} \tau, \\
& p_{e}=-\frac{A^{*}}{2 \pi U^{*}} \int_{0}^{T} C_{z}^{a}(\alpha) \sin \tau \mathrm{d} \tau .
\end{aligned}
$$




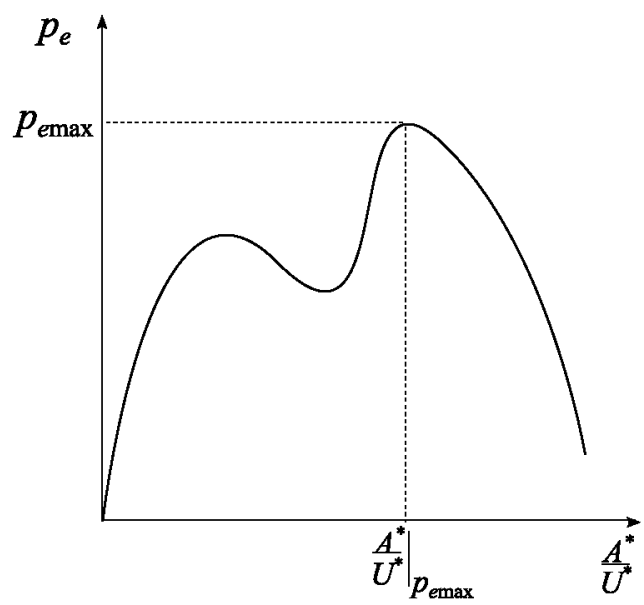

Fig. 2. Variation of the dimensionless extracted power averaged per cycle, $p_{e}$, with the relative amplitude of the motion, $A^{*} / U^{*} . A^{*} /\left.U^{*}\right|_{p_{\text {enax }}}$ is the relative amplitude that provides maximum extracted power.

From the expression of the transverse speed (7) it follows that

$$
\begin{aligned}
& \sin \tau=-\frac{1}{A \omega_{n}} \frac{\mathrm{d} z_{0}}{\mathrm{~d} t}=-\frac{U}{b \omega_{n}} \frac{b}{A} \frac{1}{U} \frac{\mathrm{d} z_{0}}{\mathrm{~d} t}=-\frac{U^{*}}{A^{*}} \tan \alpha, \\
& C_{z}^{a}(\alpha)=C_{z}^{a}(\tan \alpha)=C_{z}^{a}\left(-\frac{A^{*}}{U^{*}} \sin \tau\right)=-C_{z}^{a}\left(\frac{A^{*}}{U^{*}} \sin \tau\right),
\end{aligned}
$$

where $A^{*} / U^{*}=A \omega_{n} / U=\tan \alpha_{\operatorname{maxc}}$ is the maximum amplitude of the transverse speed during a period of oscillation divided by the velocity of the incident flow. Hence $\alpha_{\operatorname{maxc}}$ is the maximum pitch angle of velocity (or angle of attack at condition $\theta_{0}=0$ ) during one oscillation period. Considering $C_{z}^{a}(\tau)$, defined in (19), as a sinus series expansion

$$
C_{z}^{a}\left(\tau, \frac{A^{*}}{U^{*}}\right)=\sum_{n=1}^{N} b_{n} \sin n \tau ; \quad b_{n}\left(\frac{A^{*}}{U^{*}}\right)=\frac{1}{\pi} \int_{0}^{2 \pi} C_{z}^{a}\left(\tau, \frac{A^{*}}{U^{*}}\right) \sin n \tau \mathrm{d} \tau .
$$

The integral in (16) is the term $b_{1}$ of the series $C_{z}^{a}\left(\tau, A^{*} / U^{*}\right)$ in (20). Eqs. (18) and (19) show that varying $\tau$, the argument of $C_{z}^{a}$ varies sinusoidally between $-\tan \alpha_{\max }$ and $\tan \alpha_{\max }$ during the period of oscillation.

On one hand, using $\tan \alpha$ is inconvenient to fit $C_{z}^{a}(\tan \alpha)$. Note that $\tan ^{n} \alpha$ is not an orthonormal function and adding terms to the series expansion would not simplify much the study of the convergence. On the other hand, a Fourier series expansion as in (20) fits $C_{z}^{a}\left(\tau, A^{*} / U^{*}\right)$ uniformly, because every term is orthonormal to the others, and the contribution of every harmonic term of the series to the total uncertainty has a clear interpretation. In any case, the paper is focused on the extracted power, which is proportional to the term $b_{1}$ in (17), that is

$$
p_{e}=\frac{1}{2} \frac{A^{*}}{U^{*}} b_{1} .
$$

Following the procedure that leads to (14), in order to find the variation of $A^{*} / U^{*}$ with the parameter $m^{*} \zeta / U^{*}$, expressions (8) and (21) are used to impose the condition of equilibrium $p_{e}=p_{d}$

$$
\frac{1}{2} \frac{A^{*}}{U^{*}} b_{1}=\frac{2 m^{*} \zeta}{U^{*}}\left(\frac{A^{*}}{U^{*}}\right)^{2}
$$

as $b_{1}=b_{1}\left(A^{*} / U^{*}\right)$, the solution is the value of $A^{*} / U^{*}$ that satisfies

$$
\frac{4 m^{*} \zeta}{U^{*}} \frac{A^{*}}{U^{*}}=b_{1} \text {. }
$$

Note the similarity with condition (14). The maximum of the curve $p_{e}\left(A^{*} / U^{*}\right)$ appears at $\left(A^{*} / U^{*}\right)_{p_{e m a x}}$, as is shown in Fig. 2.

The value of $m^{*} \zeta / U^{*}$ that leads to $\left(A^{*} / U^{*}\right)_{p_{\text {emax }}}$ is obtained from expression (22)

$$
\left(\frac{m^{*} \zeta}{U^{*}}\right)_{p_{\operatorname{emax}}}=\frac{1}{4} b_{1}\left[\left(\frac{A^{*}}{U^{*}}\right)_{p_{\max }}\right]\left(\frac{A^{*}}{U^{*}}\right)_{p_{\operatorname{emax}}}^{-1}
$$

Once the value of $\left(m^{*} \zeta / U^{*}\right)_{p_{\operatorname{emax}}}$ is known, the mechanical design should be oriented to find the combination of values of mass, damping coefficient, natural frequency and flow speed that matches it. 
To study the different types of solutions, the extracted and dissipated power per maximum relative amplitude $\left(A^{*} / U^{*}\right)$ in a cycle (or specific power) can be used:

$$
\tilde{p}_{e}=\frac{p_{e}}{\frac{A^{*}}{U^{*}}}=\frac{1}{2} b_{1}, \quad \tilde{p}_{d}=\frac{p_{d}}{\frac{A^{*}}{U^{*}}}=\frac{2 m^{*} \zeta}{U^{*}}\left(\frac{A^{*}}{U^{*}}\right),
$$

so that the shape of the curve $b_{1}\left(A^{*} / U^{*}\right)$ determines the possible solutions. The equilibrium points fulfil the condition $b_{1}=2 \tilde{p}_{d}$.

As shown in Fig. 3a, there are multiple scenarios possible as the slope of $\tilde{p}_{d}$ is increased. The labels in the curves show the different cases: (1) one solution, (2) three solutions (two stable and one unstable) and (3) limit case.

Studying the shape of the curves $b_{1}$ (independently of the dissipation), if no inflection point exists, the solution is unique and stable. If an inflection point appears in the increasing part of the curve, there are two solutions, one stable and another one unstable (Fig. 3b, case 2 ). If the complexity of the shape of the curve $b_{1}\left(A^{*} / U^{*}\right)$ increases, a different behaviour can appear.

The dimensionless dissipated power averaged per cycle and per maximum relative amplitude, $\tilde{p}_{d}$, defined in (24), is a linear function of $\left(A^{*} / U^{*}\right)$, so that if $U^{*}$ is small or $m^{*} \zeta$ is big, the slope of $\tilde{p}_{d}$ increases and a limit, in which no stable solution exists, is reached (Fig. 3, case 3 ).

The determination of the maximum dissipated or extracted power from the experimental results is defined by the maximum of the curve $p_{e}\left(A^{*} / U^{*}\right)=p_{e}\left(\left(A^{*} / U^{*}\right)_{p_{\text {emx }}}\right)$ obtained from (21). The curves of maximum power as a function of the pitch angle of the body, $\theta$, can be obtained as shown in Fig. 4. From the maximum dimensionless extracted power, $p_{\text {emax }}$, for different pitch angles of the body, the curve of maximum efficiency can be determined as a function of the pitch angle of the body (Fig. 4).

The main advantage of this method is that $b_{1}\left(A^{*} / U^{*}\right)$ can be calculated numerically using (20) directly from experimental results without polynomial fitting, which could be of a high order depending on the shape of the curve. In such a case, the possible advantages of an analytical treatment are lost.

\subsection{Comparison between numerical integration method and polynomial expansion}

To validate the numerical integration method and to estimate the influence of the parameters involved in the uncertainty of the results, the polynomial expressions given in Barrero-Gil et al. (2009) have been used as reference curves of
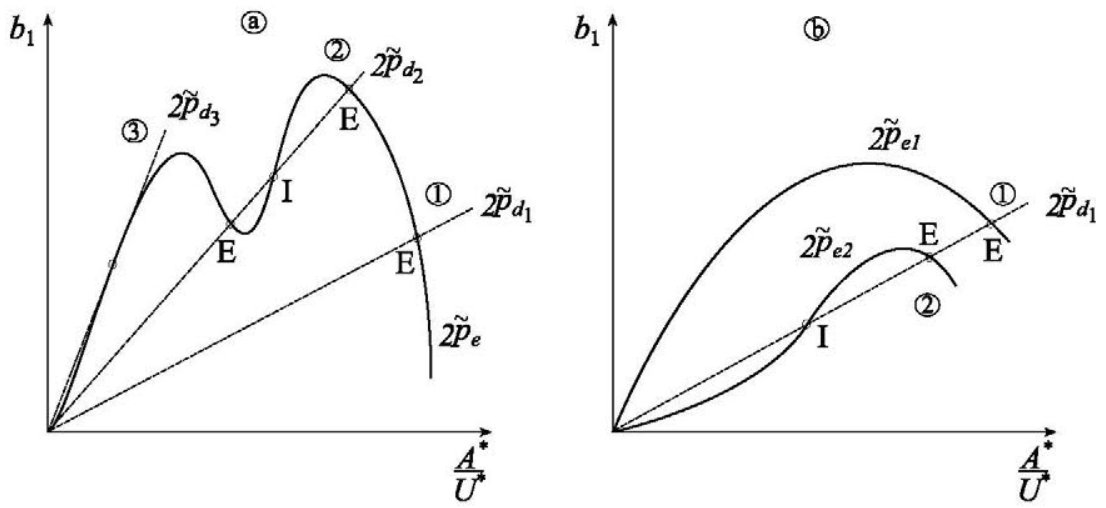

Fig. 3. Variation of $b_{1}$ with the relative amplitude $A^{*} / U^{*}$. (a) Possible equilibrium solutions: (1) one solution, (2) 3 solutions, (3) limit case. E: stable, I: unstable. (b) No inflection points (case 1); one inflection point (case 2).
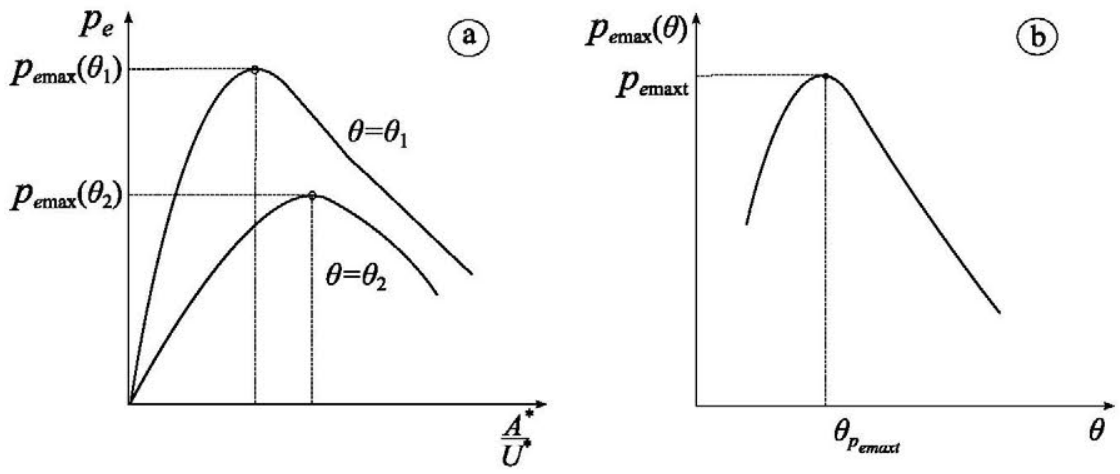

Fig. 4. Determination of the maximum dimensionless extracted power from experimental results. (a) Variation of dimensionless extracted power, $p_{e}$, as a function of relative amplitude, $A^{*} / U^{*}$, for two values of the pitch angle $\theta$. (b) Variation of maximum extracted power, $p_{\text {emax }}$, with the pitch angle of the body, $\theta$. 
dimensionless coefficient of transverse force $C_{z}$ over the body as follows:

$$
C_{z}(\alpha)=a_{1} \tan \alpha+a_{3} \tan ^{3} \alpha+a_{5} \tan ^{5} \alpha+a_{7} \tan ^{7} \alpha .
$$

The coefficients of the polynomials used for validating the method presented here to analyse the galloping stability are shown in Table 1.

An analytic expression for $b_{1}$ can be obtained from (25) using definition (20) or (12), as follows:

$$
b_{1}=b_{1 a}=\frac{1}{2} a_{1}\left(\frac{A^{*}}{U^{*}}\right)+\frac{3}{8} a_{3}\left(\frac{A^{*}}{U^{*}}\right)^{3}+\frac{5}{16} a_{5}\left(\frac{A^{*}}{U^{*}}\right)^{5}+\frac{35}{128} a_{7}\left(\frac{A^{*}}{U^{*}}\right)^{7} \text {. }
$$

The formula integrated to obtain $b_{1 n}$ is

$$
b_{1}=b_{1 n}=\frac{1}{\pi} \int_{0}^{2 \pi} C_{z}\left(\tau, \frac{A^{*}}{U^{*}}\right) \sin (\tau) \mathrm{d} \tau .
$$

The results from the numerical method $b_{1 n}$ are compared in Fig. 5 with the analytic expression, $b_{1 a}$, (26). The data $C_{z}(\alpha)$ are discretized at an interval $\Delta \alpha=1^{\circ}$.

\section{Table 1}

Polynomial coefficients used in the validation of the numerical integration method, extracted from Barrero-Gil et al. (2009) for different cases $k$.

\begin{tabular}{llrll}
\hline$k$ & $a_{1}$ & $a_{3}$ & $a_{5}$ & $a_{7}$ \\
\hline 1 & 8 & -75 & 0 & 0 \\
2 & 1.5 & 231 & -3800 & 0 \\
3 & 4.72 & -294 & 10972 & -105000 \\
4 & 2.7 & -168 & 6270 & -60000 \\
\hline
\end{tabular}

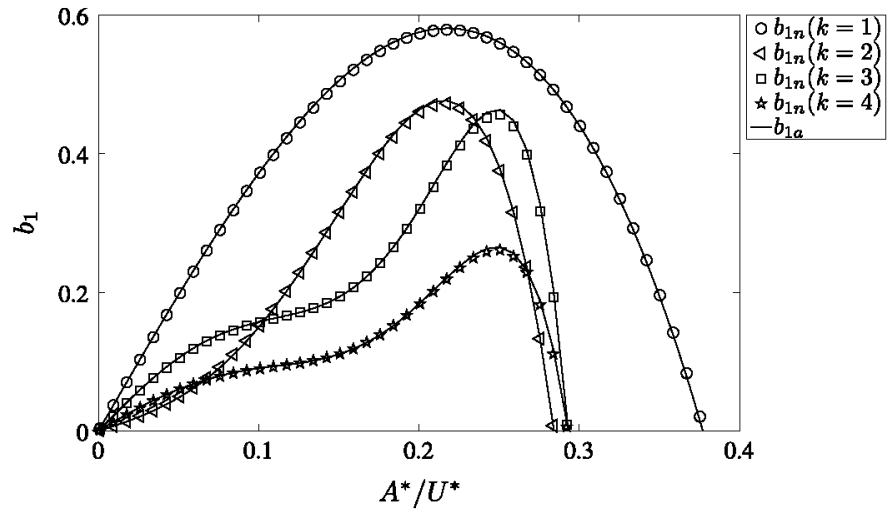

Fig. 5. Variation of coefficient, $b_{1}$, with the relative amplitude, $A^{*} / U^{*}$. Comparison of the analytical solution, $b_{1 a}$, and the numerical integration, $b_{1 n}$, for different curves of $C_{z}$ (polynomial functions) around $\alpha=0^{\circ}$, for the cases $k$ according to Table 1 . Discretization $\Delta \alpha=1^{\circ}$.

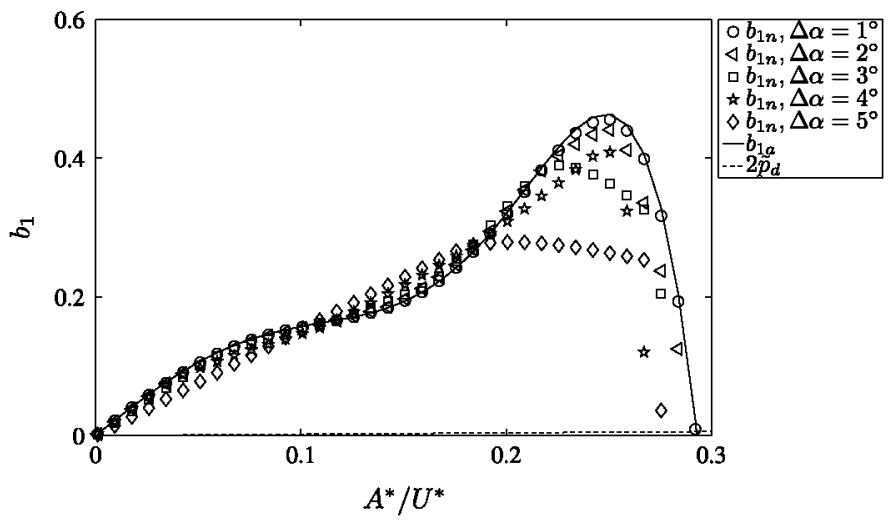

Fig. 6. Variation of coefficient, $b_{1}$, with the relative amplitude, $A^{*} / U^{*}$. Influence of the discretization interval, $\Delta \alpha$, of the force coefficient, $C_{z}$, in the numerical integration of $b_{1}$. Case $k=3$ of Table 1 . 
The good agreement between the analytical expression $b_{1 a}$ and the numerical integration $b_{1 n}$ can be appreciated in Fig. 5 for all the cases that were considered.

\subsection{Influence of the number of discretization points}

The number of measurement points required in order to properly characterize the dimensionless coefficient curve of the transverse force, $C_{z}$, needs to be determined so that integration (27) can be made with an acceptable error. A study of the influence of the discretization is performed for $\Delta \alpha=1^{\circ}, 2^{\circ}, 3^{\circ}, 4^{\circ}, 5^{\circ}$ (see Fig. 6) in the polynomial curve $k=3$ from Table 1 . In this case, the experimental data $c_{l}(\theta), c_{d}(\theta)$ are considered, and the simulated experimental force coefficient $C_{z}(\alpha)$ is taken directly from (26) at pitch intervals $\Delta \alpha=\Delta \theta$.

The specific dissipation power, $\tilde{p}_{d}$, used in Fig. 6, has been estimated from the results reported in Alonso et al. (2012), and also from the wind tunnel tests performed at IDR/UPM with similar bodies (see Table 2).

The maximum of the curve $b_{1}$ in Fig. 6 corresponds to twice the maximum specific extracted power, $\tilde{p}_{\text {emax }}$.

In order to estimate the uncertainty due to the discretization of the curve $C_{z}(\alpha)$, the parameter $\varepsilon_{\text {disc }}$ is defined as

$$
\varepsilon_{\text {disc }}=\left|\frac{\left.\frac{A^{*}}{U^{*}}\right|_{\text {eqa }}-\left.\frac{A^{*}}{U^{*}}\right|_{\text {eqn }}}{\left.\frac{A^{*}}{U^{*}}\right|_{\text {eqa }}}\right|
$$

where $A^{*} /\left.U^{*}\right|_{\text {eqa }}$ is the relative amplitude at the equilibrium for a certain dissipation which is obtained analytically from condition (14) and the analytic expression of $b_{1} . A^{*} /\left.U^{*}\right|_{\text {eqn }}$ is the relative amplitude at the equilibrium which is obtained from condition (14) and the numerical integration of $b_{1}$.

As shown in Fig. 7, for a discretization interval $\Delta \alpha=1^{\circ}$, the uncertainty due to discretization is under $0.3 \%$, and around $10 \%$ for a discretization interval $\Delta \alpha=5^{\circ}$.

\subsection{Comparison of the numerical integration method with the polynomial expansion at a point different from the origin}

In the process of validation of the numerical integration of $b_{1}$ in the study of transverse galloping, the case of a body at a pitch angle different from the origin $\left(\theta=\theta_{0} \neq 0\right)$ was considered. A polynomial of third degree has been considered (case $k=1$, see Fig. 8), where the coefficients are the first row in Table 1 . To calculate the value of the analytic expression of $b_{1}$, $b_{1 a}$, in the offset galloping ( $\alpha=\theta_{0}+\beta, \theta_{0}$ being the new angle origin), the expression of $C_{z}$ has been expressed in terms of $\beta^{n}$, and $b_{1}$ calculated analytically:

$$
C_{z}(\beta)=a_{0}+a_{1} \theta_{0}+a_{3} \theta_{0}^{3}+\left(a_{1}+3 a_{3} \theta_{0}^{2}\right) \beta+3 a_{3} \theta_{0} \beta^{2}+a_{3} \beta^{3},
$$

Table 2

Values used to estimate the specific dissipation power, $\tilde{p}_{d}$, extracted from Alonso et al. (2012).

\begin{tabular}{ll}
\hline Characteristic transverse dimension of the body, $b(\mathrm{~m})$ & $0.1-0.3$ \\
Structural damping, $\zeta$ & 0.014 \\
Natural frequency, $\omega_{n} / 2 \pi(\mathrm{Hz})$ & 2.5 \\
Mass, $m(\mathrm{~kg} / \mathrm{m})$ & 2.65 \\
Dimensionless velocity, $U^{*}=U /\left(\omega_{n} b\right)$ & 7.64 \\
\hline
\end{tabular}

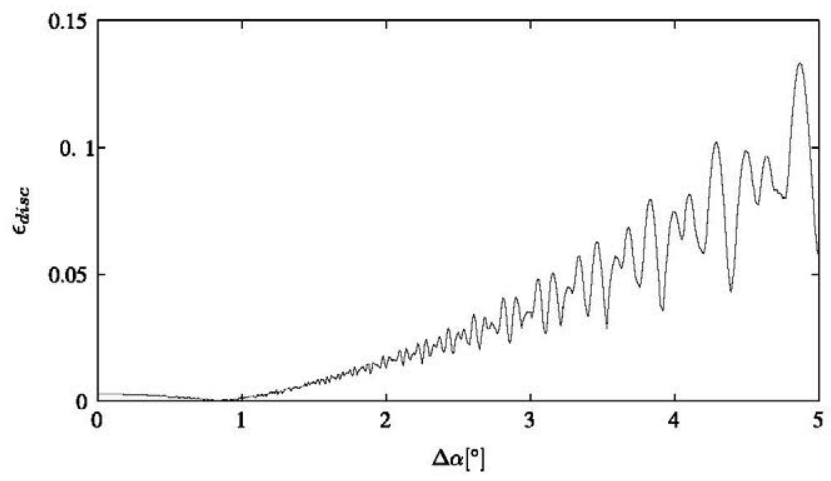

Fig. 7. Variation of the uncertainty in the relative amplitude at the equilibrium, $\varepsilon_{\text {disc }}$, as a function of the discretization interval $\Delta \alpha$ of the curve $C_{z}(\alpha)$. Case $k=3$ of Table 1 . 
so that the new coefficients to be used in the expression of $b_{1 a}(26)$ are

$$
\begin{aligned}
& a_{3}^{\prime}=a_{3} \\
& a_{2}^{\prime}=3 a_{3} \theta_{0} \\
& a_{1}^{\prime}=a_{1}+3 a_{3} \theta_{0}^{2} \\
& a_{0}^{\prime}=a_{0}+a_{1} \theta_{0}+a_{3} \theta_{0}^{3}
\end{aligned}
$$

and from (26), $b_{1 a}$ can be determined as

$$
b_{1 a}=\frac{1}{2} a_{1}^{\prime}\left(\frac{A^{*}}{U^{*}}\right)+\frac{3}{8} a_{3}^{\prime}\left(\frac{A^{*}}{U^{*}}\right)^{3} \text {. }
$$

From the new displaced curve, $C_{z d}$, the antisymmetric part $C_{z d a}$ is extracted to proceed with the integration.

Fig. 9 shows that by increasing the pitch angle $\theta_{0}$ the shape of the curve $C_{z d a}$ changes till the slope at the origin $\alpha=0$ is horizontal (at $\theta_{0}=0.2 \mathrm{rad}$ ), and then galloping does not appear.

The comparison between the results from numerical integration $b_{1 n}$ and the analytic solution $b_{1 a}(30)$ at different values of pitch angle $\theta_{0}$ is shown in Fig. 10, for a discretization interval $\Delta \theta=1^{\circ}$. A good agreement between both solutions can be appreciated, as numerical and analytical results cannot be distinguished. However, a small difference between both solutions can be noticed in Fig. 11, which is due to a larger discretization interval $\Delta \theta=4^{\circ}$. The solid lines next to each numerical solution, $b_{1 n}$, are the respective analytical solutions, $b_{1 a}$, for each case.

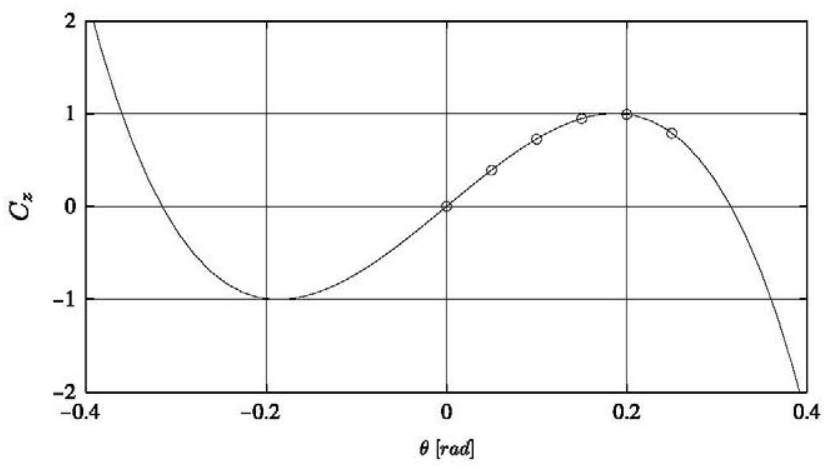

Fig. 8. Variation of the transverse force coefficient, $C_{z}$, with the pitch angle of the body, $\theta$. Symbols: pitch angles analysed in Fig. 9 . Case $k=1$, see Table 1.
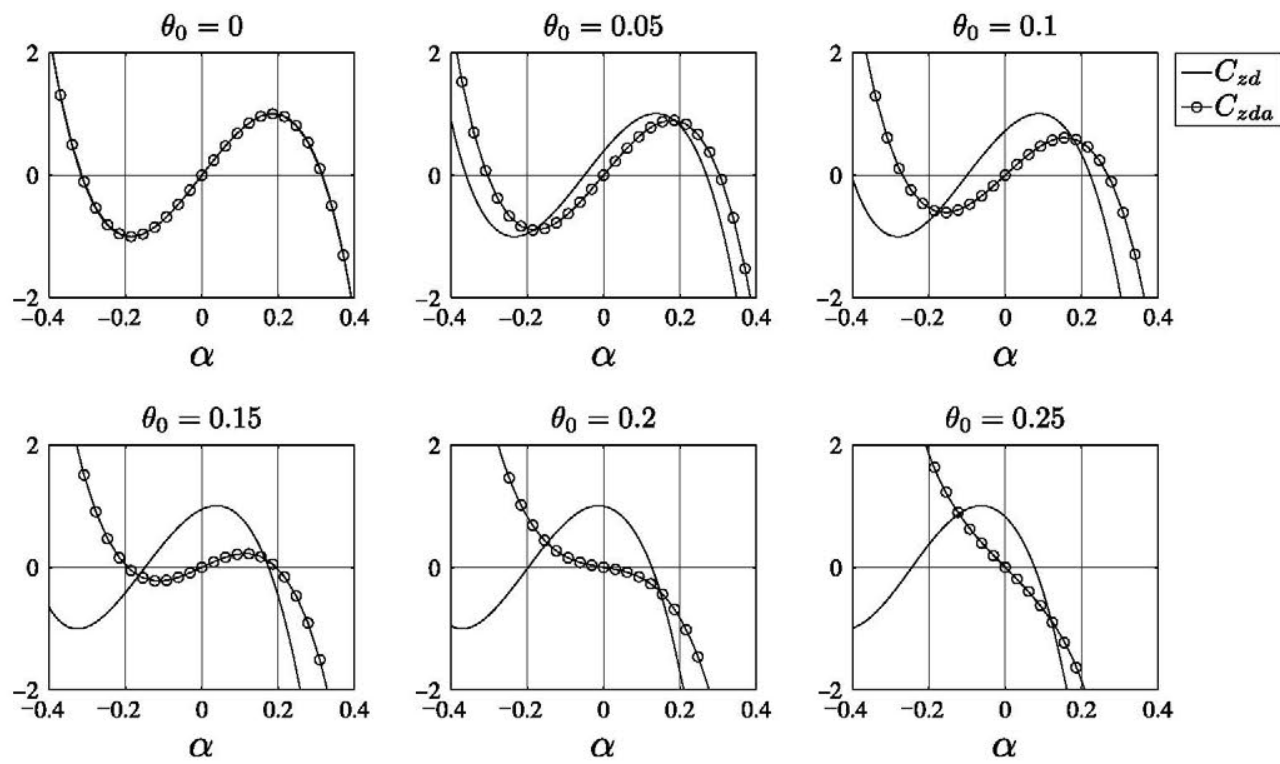

Fig. 9. Variation of the transverse force coefficient, $C_{z d}(\alpha)$, considering different pitch angles, $\theta_{0}$, and the antisymmetric function, $C_{z d a}$, used in the numerical integration of $b_{1}$. Case $k=1$, for $\theta_{0}$ angles shown in Fig. 8. 
In order to better evaluate the effect of the discretization interval of the wind tunnel measurements, the standard deviation between the analytical solution, $b_{1 a}$, and the numerical one, $b_{1 n}$, is defined as

$$
\sigma_{b 1}=\sqrt{\frac{\sum\left(b_{1 n}-b_{1 a}\right)^{2}}{N}}
$$

where $N$ is the number of samples considered $N \simeq 90$ in most of these cases.

The standard deviation, $\sigma_{b 1}$, is less than $0.5 \%$ for discretization intervals less than $\Delta \theta=2^{\circ}$ and around $4 \%$ for $\Delta \theta=5^{\circ}$, as can be seen in Fig. 12. The influence on $\sigma_{b 1}$ of the pitch angle of the body, $\theta_{0}$, is not very relevant.

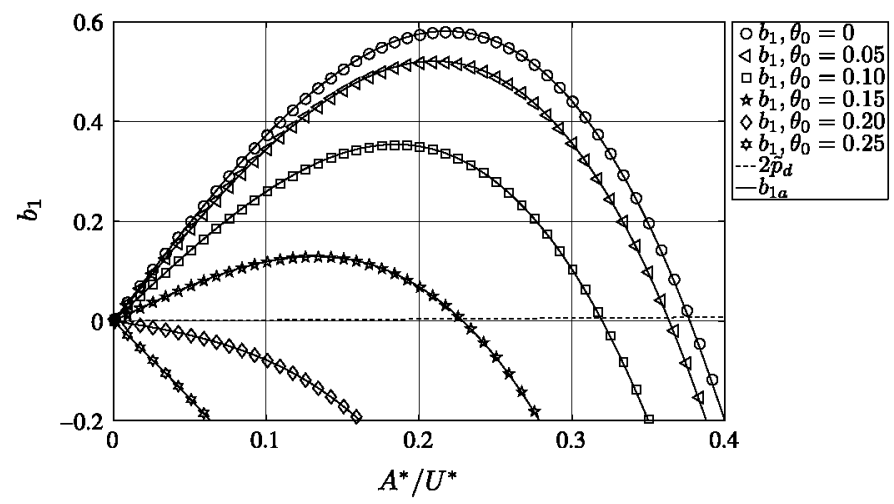

Fig. 10. Variation of coefficient $b_{1}$ with the relative amplitude $A^{*} / U^{*}$, obtained by numerical integration, $b_{1 n}$, at configurations with pitch angle $\theta_{0} \neq 0$, and $b_{1}$ analytic, $b_{1 \alpha}$. Discretization interval $\Delta \theta=1^{\circ}$. Case $k=1$ in Table 1 . For values of $\theta_{0}$ see Fig. 8 .

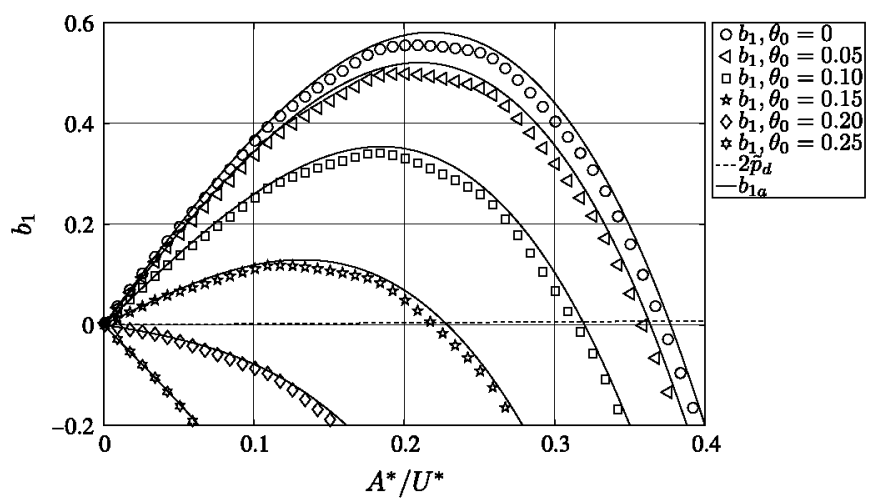

Fig. 11. Variation of coefficient $b_{1}$ with the relative amplitude $A^{*} / U^{*}$, obtained by numerical integration, $b_{1 n}$, at configurations with pitch angle $\theta_{0} \neq 0$, and analytical solution, $b_{1 a}$. Discretization interval $\Delta \theta=4^{\circ}$.

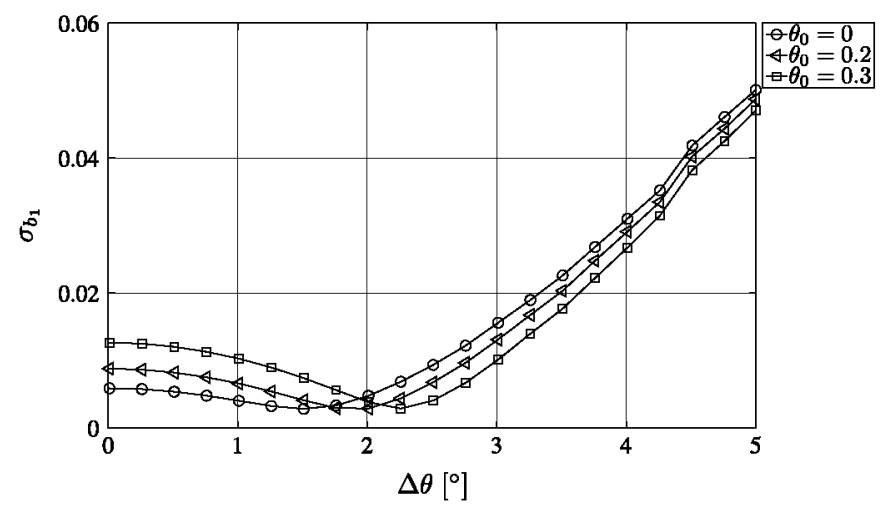

Fig. 12. Variation with the discretization interval $\Delta \theta$ of the standard deviation, $\sigma_{b 1}$, between the analytic solution $b_{1 a}$ and the numerical integration $b_{1 n}$ due to the discretization of $C_{z}$ for different pitch angles $\theta_{0}$. Case $k=1$ in Table 1 . 


\section{Experimental set-up}

To explain the application of the method to some practical cases, measurements have been performed in two different wind tunnels, A4C and A9, both properties of IDR/UPM.

The $\mathrm{A} 4 \mathrm{C}$ wind tunnel is an open-circuit, closed-test-section wind tunnel with a two-dimensional contraction. The dimensions of its test chamber are length $1.2 \mathrm{~m}$, width $0.2 \mathrm{~m}$ and height $1.8 \mathrm{~m}$. The diffuser behind the test chamber expands and adapts the flow generated by four centrifugal blowers, each of $7.5 \mathrm{~kW}$. The return circuit is the reduced space between the wind tunnel and the walls of the room. The wind speed profile at the model test section was uniform within $\pm 1 \%$ and the turbulence intensity was around $7 \%$. The test bodies were positioned using a NEWPORT EPS100 rotating platform, which allows the angle of attack of the airfoil to be set with an uncertainty of $\pm 0.1^{\circ}$. The blockage of the A4C wind tunnel was around $10 \%$ for the biconvex cross-section at $\theta=72^{\circ}$.

The aerodynamic loads were measured with an ATI six-component strain-gauge balance, model Gamma SI-130-10. An AirFlow 048 Pitot tube was located at the entrance of the test chamber and was connected to a Druck LPM5480 pressure transducer.

The A9 wind tunnel is also an open-circuit, closed-test-section wind tunnel with a two-dimensional contraction. The dimensions of the test chamber are length $2.0 \mathrm{~m}$, width $1.5 \mathrm{~m}$ and height $1.8 \mathrm{~m}$. The aerodynamic loads were measured with an ATI six-component strain-gauge balance, model Delta FT5575. The blockage of the A9 wind tunnel was in the range 3-9\% for the rhomboidal cross-section at $\theta=0^{\circ}$ and $\theta=90^{\circ}$.

The wind speed vertical profile at the model test section was uniform within $\pm 1 \%$ and the turbulence intensity was around 2.5\%. The Pitot tube was connected to a Druck LPM9381 pressure transducer.

In both wind tunnels the sampling frequency used was $200 \mathrm{~Hz}$, as the maximum frequency in the flow is that of the vortex shedding, $f_{v}$, which calculated from the Strouhal number $\left(\mathrm{St}=f_{v} D / U\right)$ was under $20 \mathrm{~Hz}$.

In the case of the rhomboidal cross-section, the value of the parameters are $D=0.11 \mathrm{~m}$ (characteristic length of the structure transverse to the incident flow), $U=14.5 \mathrm{~m} / \mathrm{s}$ (velocity of the flow in the A9 wind tunnel test chamber) and St $\sim 0.15$, and the measuring period was $30 \mathrm{~s}$.

Note that measurements in both tunnels have not been corrected for blockage. This effect has not been taken into account due to the fact that the objective of this paper is to show the feasibility of the proposed analysis method.

\section{Results and discussion}

In this section the study of the stability for several cylindrical bodies with different cross-section shapes is performed, by comparing the relative position of the curves of extracted and dissipated power. It is based on the integration of the curves $C_{z}$ obtained from the experimental results measured with a balance in a wind tunnel. The cross-section shapes analysed were biconvex, D-shape, rhomboidal of different relative thicknesses, triangles extracted from Alonso et al. (2012) and the square extracted from $\mathrm{Ng}$ et al. (2005) and Parkinson and Smith (1964).

The pitch angle intervals at which the body is prone to galloping are determined using the Glauert-Den Hartog criterion $H=\partial c_{l}(\alpha) / \partial \alpha+c_{d}(\alpha)<0$. The mechanical properties can be synthesized by the Scruton number Sc, defined as

$$
\mathrm{Sc}=\frac{2 m \zeta}{\rho b^{2}}
$$

so that from the values in Table 2, it can be deduced that the Scruton number is between 1 and 7 depending on the characteristic transverse dimension of the body. The values of these mechanical properties, along with the stiffness of the spring, have been chosen so that the body motion speed is slow relative to the speed of a fluid particle, and then the hypothesis of quasi-stationary aerodynamic forces can be justified.

In all the cases presented hereafter, the discretization interval was $\Delta \theta=1^{\circ}=0.017 \mathrm{rad}$.

\subsection{Biconvex airfoil analysis}

After studying some biconvex airfoils of different relative thicknesses in the $\mathrm{A} 4 \mathrm{C}$ wind tunnel (measuring global forces with a balance), it was observed that the airfoil of relative thickness $E_{r}=b / c=0.817$ (where $b$ is the characteristic transverse dimension and $c$ the chord of the airfoil) showed the greatest galloping intensity (Alonso et al., 2009). The pitch angles were measured with regard to the plane of symmetry of the airfoil, as is shown in Fig. 13. The Reynolds number during the measurements, based on the chord of the airfoil $c=0.2 \mathrm{~m}$, was $\operatorname{Re}=2.01 \times 10^{5}$.

The results from the measurements of $c_{b}, c_{d}$, and the Glauert-Den Hartog criterion, $H$, are shown in Fig. 14, where a region around $\theta_{0} \sim 70^{\circ}=1.22 \mathrm{rad}$ can be appreciated in which the necessary condition for galloping, $H<0$, is satisfied.

Fig. 15 shows the force coefficient, $C_{z}$, obtained from the experimental data using (2) for different values of the pitch angle of the body, $\theta_{0}$. The origin of the pitch angles, $\theta_{0}$, was displaced around $\theta=70^{\circ}=1.22 \mathrm{rad}$, and the displaced data were interpolated, $C_{z z}$, with a discretization interval $\Delta \theta=1^{\circ}=0.017 \mathrm{rad}$. A linear interpolation - a Matlab function - was used.

The possible points of equilibrium for the biconvex airfoil, for different pitch angles of the body $\theta_{0}$, are placed at the crossing points of the $b_{1}$ curves with the $2 \tilde{p}_{d}$ dissipation line (see Fig. 16). At $\theta_{0} \sim 76^{\circ}=1.33$ rad a limit case is reached and the existence of an equilibrium solution is no longer possible. 


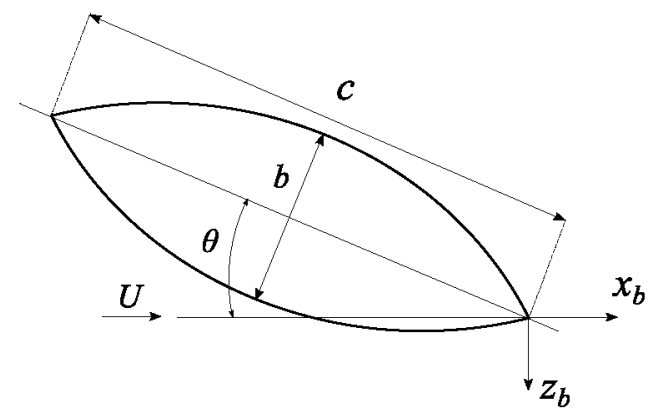

Fig. 13. Nomenclature in the case of a biconvex airfoil.
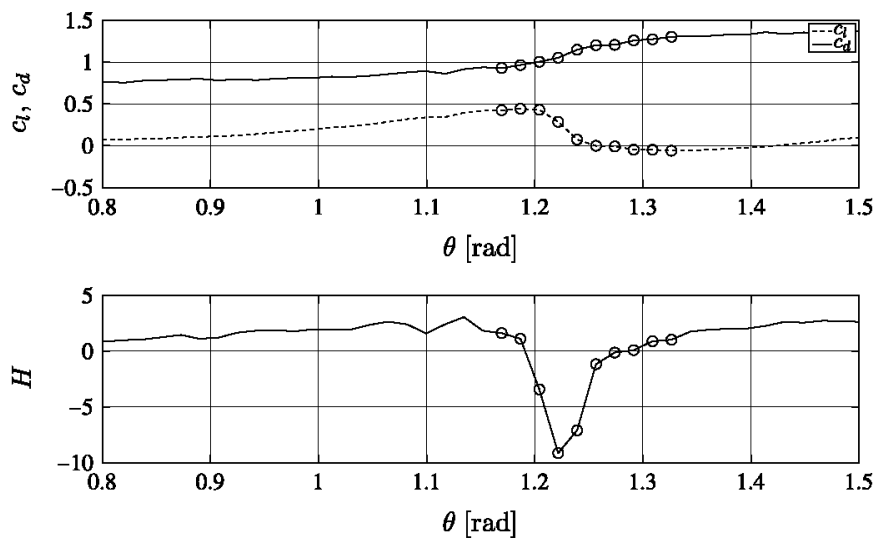

Fig. 14. Variation with the pitch angle of the body, $\theta$, of the coefficients of lift, $c_{l}$, and drag, $c_{d}$, and of the Glauert-Den Hartog parameter, $H$, over the biconvex airfoil of relative thickness $E_{r}=0.817$ in the $A 4 C$ wind tunnel. Symbols indicate the pitch angles $\theta_{0}$ considered in the analysis of Figs. 15 and 16 .
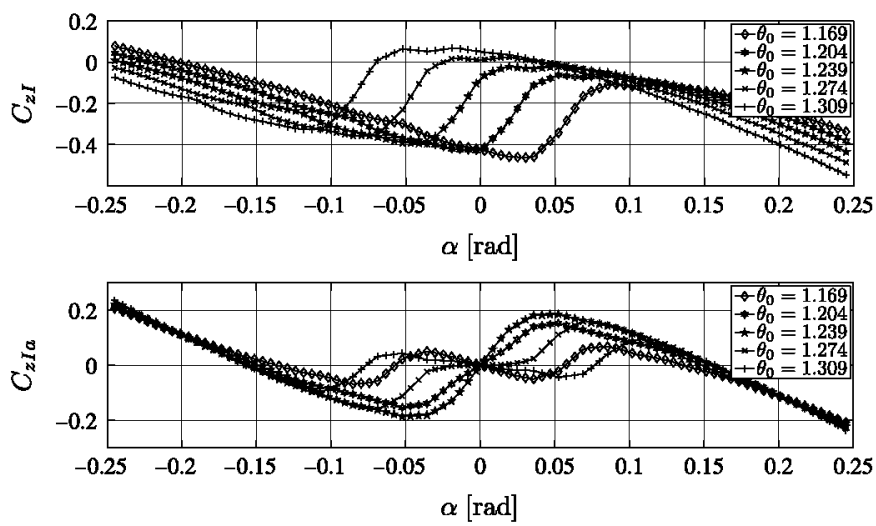

Fig. 15. Variation of the force coefficient, $C_{2}$, with the angle of attack, $\alpha$, where $\alpha=\theta-\theta_{0}$, for different values of pitch angle $\theta_{0}$, around $\theta_{0}=70^{\circ}=1.221$ rad. Curve $C_{z}$ interpolated, $C_{z J}$, and its antisymmetric part, $C_{z J a}$, for the biconvex airfoil of relative thickness $E_{r}=0.817$.

In Fig. 16 it can also be seen that, for the dissipation chosen, a stable solution exists in the range $1.169 \leq \theta_{0} \leq 1.326$ rad with a relative amplitude of oscillation $0.15<A^{*} / U^{*}<0.19$; besides this stable solution, there is one unstable solution in the range $1.169 \leq \theta_{0} \leq 1.187 \mathrm{rad}$, and another in the range $1.292<\theta_{0}<1.326 \mathrm{rad}$.

For a given dissipated power, $\tilde{p}_{d}$, there is a pitch angle, $\theta$, which provides a maximum relative amplitude of equilibrium $A^{*} /\left.U^{*}\right|_{e q}$, as shown in Fig. 17.

Another approach is to use the dissipation as a parameter, so that for every pitch angle, $\theta$, there is a specific dissipated power, $\tilde{p}_{d}$ (the straight line (24)), which maximizes the specific extracted power, $\tilde{p}_{e}$. The maximum values of $\tilde{p}_{e}, \tilde{p}_{\text {emax }}$ as a function of $\theta$ are shown in Fig. 18. Note that, despite the fact that the maximum of the relative amplitude is reached at $\theta \sim 71.62^{\circ}=1.25 \mathrm{rad}$ (for a certain dissipated power), the maximum specific dissipated power, $\tilde{p}_{d}$ (and the extracted, $\tilde{p}_{e}$ ), is obtained for $\theta=71.05^{\circ}=1.24 \mathrm{rad}$, although in the range $1.22<\theta<1.25$ rad the dissipated power is close to the maximum value. 


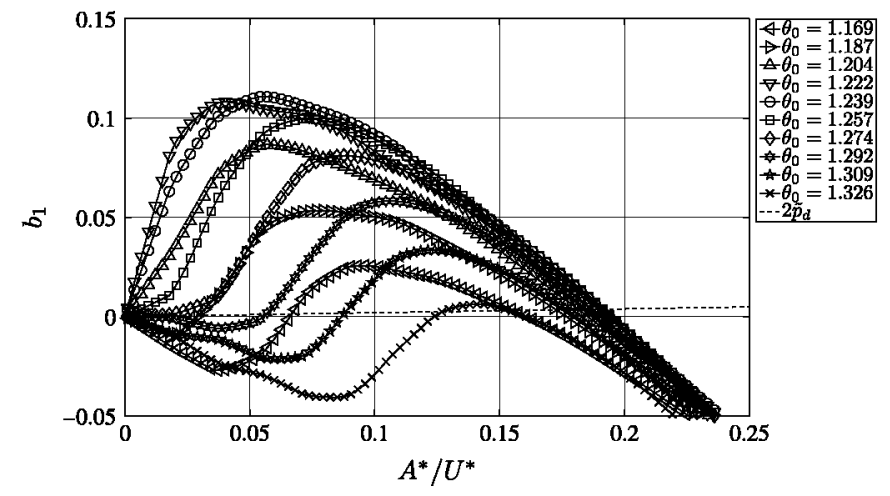

Fig. 16. Variation with the relative amplitude, $A^{*} / U^{*}$, of the coefficient $b_{1 n}$ from the numerical integration, and the specific dissipated energy, $\tilde{p}_{d}$, for a biconvex airfoil of relative thickness $E_{r}=0.817$ oscillating around a pitch angle of the body $\theta_{0}=1.221 \mathrm{rad}=70^{\circ}$.

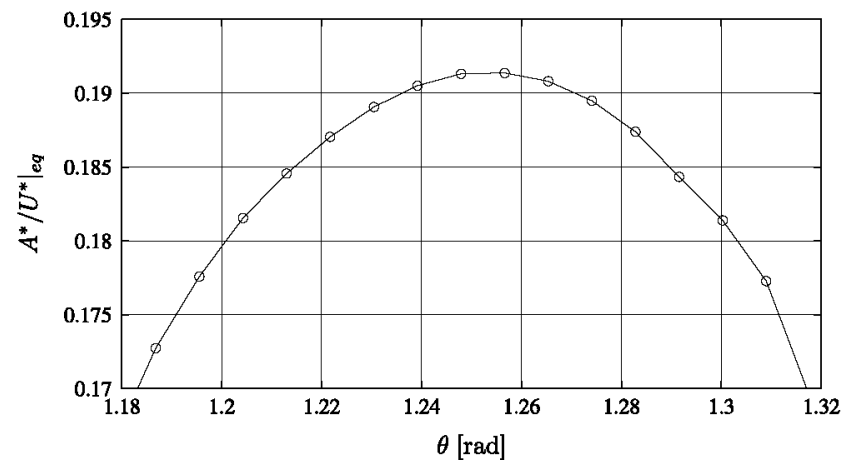

Fig. 17. Variation of the relative amplitude of equilibrium, $A^{*} /\left.U^{*}\right|_{e q}$, as a function of the pitch angle, $\theta$, for a certain specific dissipated power, $\tilde{p}_{d}$, obtained from the parameters in Table 2.

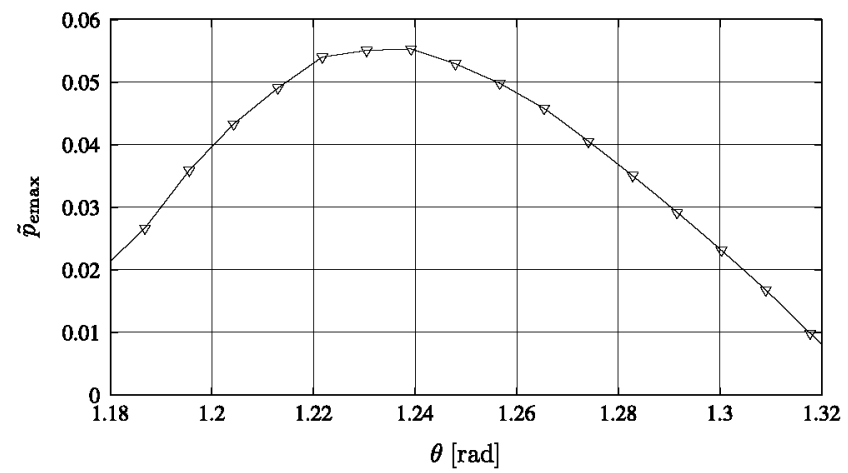

Fig. 18. Variation of the maximum specific extracted power, $\tilde{p}_{\text {emax }}=\left.\frac{1}{2} b_{1}\right|_{\max }$, as a function of the pitch angle of the body, $\theta$.

In Fig. 19 it can be observed that the dimensionless extracted power averaged per cycle, $p_{e}$, is one order of magnitude smaller than the specific extracted power due to the fact that the relative amplitude $\left(A^{*} / U^{*}\right)_{p_{\max }}$ that provides the maximum dimensionless extracted power averaged per cycle $p_{\text {emax }}$ is small compared to unity. The maximum of $p_{\text {emax }}$ appears at $\theta_{0}=1.27 \mathrm{rad}$ and the maximum of $\tilde{p}_{\text {emax }}$ appears at $\theta_{0}=1.23 \mathrm{rad}$. This could be useful for designs in which the relative amplitude could be a limiting factor.

\subsection{D-shape analysis}

The measurement tests of the D-shape cross-section body were performed in the A9 wind tunnel. The test configuration is shown in Fig. 20.

The Reynolds number during the test, based on the radius of the circular part of the $\mathrm{D}$-shape body, $b=0.126 \mathrm{~m}$, was $\operatorname{Re}=1.05 \times 10^{5}$ (Fig. 21). 


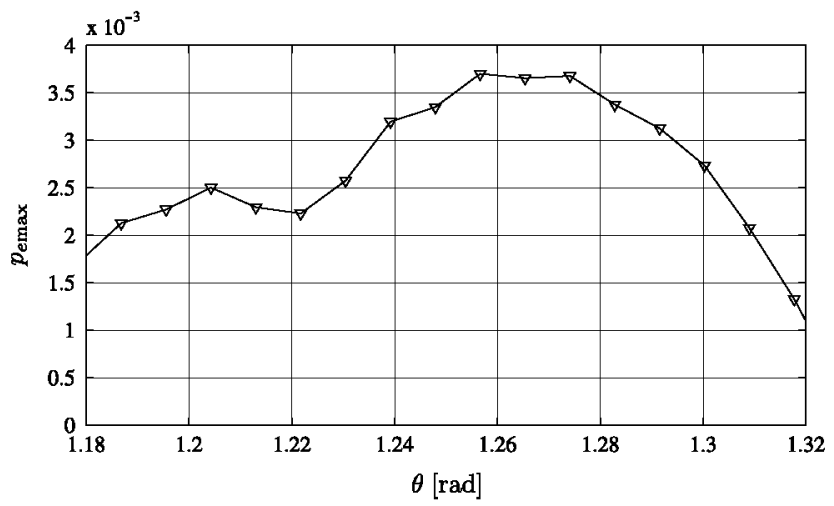

Fig. 19. Variation of the maximum dimensionless extracted power, $p_{e m a x}$, as a function of the pitch angle of the body, $\theta$.

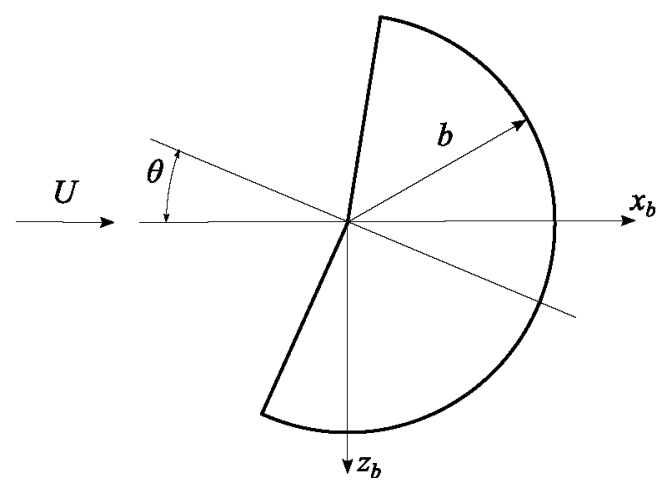

Fig. 20. Nomenclature in the case of the D-shape body.
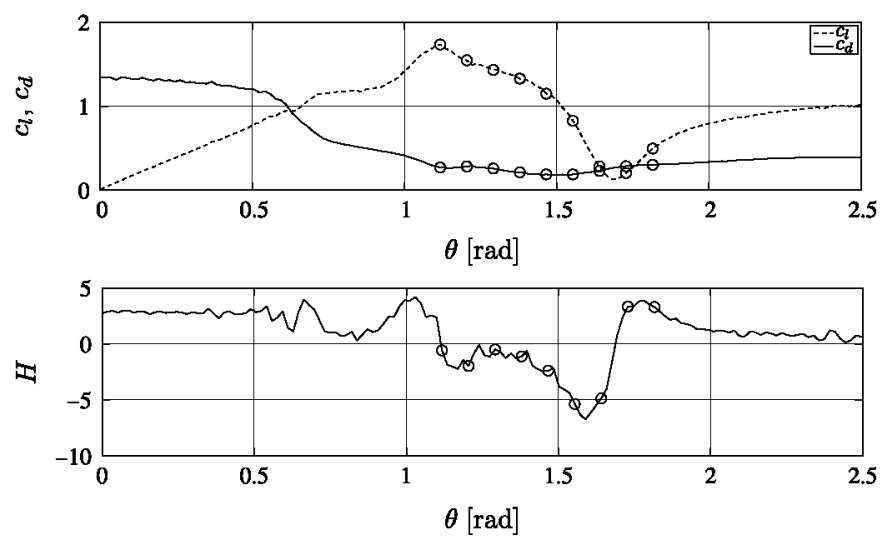

Fig. 21. Variation with the pitch angle of the body, $\theta$, of the coefficients of lift, $c_{l}$, and drag, $c_{i}$, and Glauert-Den Hartog parameter, $H$, over the D-shape body tested in the A9 wind tunnel. Symbols indicate the pitch angles $\theta_{0}$ included in the analysis (see Fig. 22).

In the results presented in Fig. 22, it can be appreciated that a stable solution appears around $\theta_{0} \sim 64^{\circ}=1.117$ rad with a relative amplitude of oscillation $A^{*} / U^{*} \simeq 0.03$ and it disappears around $\theta_{0} \sim 74^{\circ}=1.292 \mathrm{rad}$. At $\theta_{0} \sim 1.204 \mathrm{rad}$ there are three solutions: two stable solutions with a relative amplitude of oscillation $A^{*} / U^{*} \simeq 0.25$ and $A^{*} / U^{*} \simeq 0.66$ respectively, and one unstable with $A^{*} / U^{*} \simeq 0.35$. For higher pitch angles only one stable solution exists, of relative amplitude $0.7<A^{*} / U^{*}<0.9$.

Note the complex way in which the shape of the curves $b_{1}\left(A^{*} / U^{*}\right)$ varies with $\theta_{0}$, showing the existence of several types of oscillations as the pitch angle changes. 


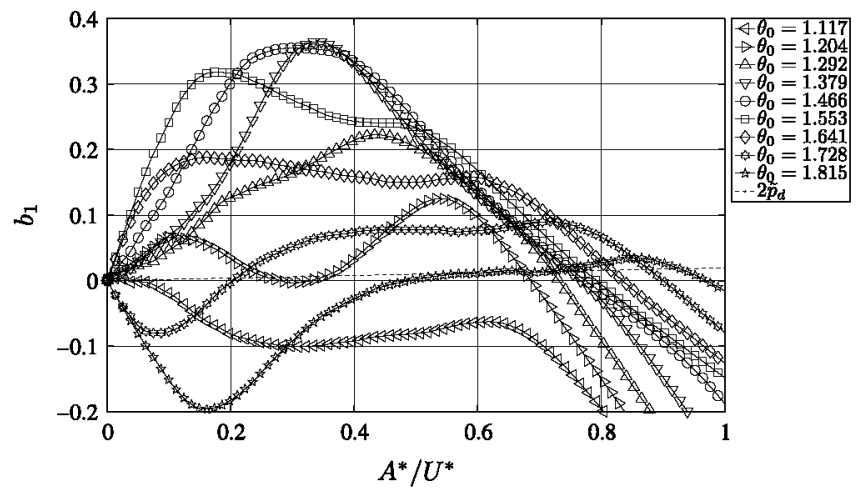

Fig. 22. Variation with the relative amplitude, $A^{*} / U^{*}$, of the coefficient $b_{1 n}$ obtained from the numerical integration and the dissipation, $\tilde{p}_{d}$, for the D-shape body, oscillating around a pitch angle of the body close to $\theta=80^{\circ}=1.4 \mathrm{rad}$.

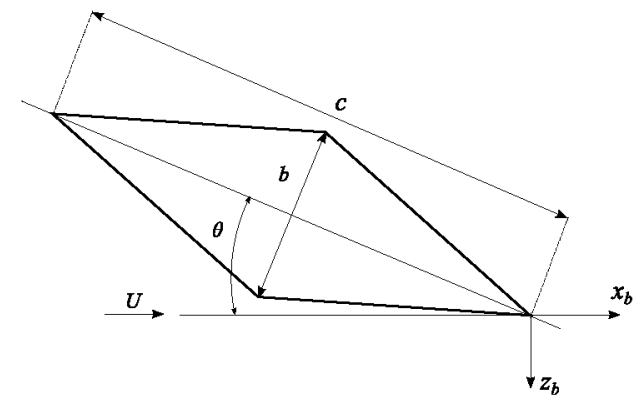

Fig. 23. Nomenclature in the case of the rhomboidal cross-section airfoil.
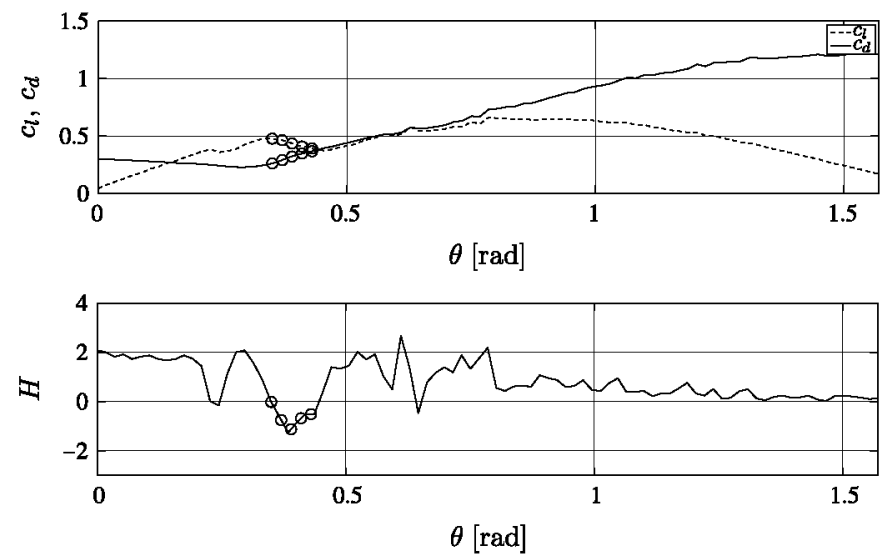

Fig. 24. Variation with the pitch angle of the body, $\theta$, of the coefficients of lift, $c_{l}$, and drag, $c_{d}$, and Glauert-Den Hartog parameter, $H$, over the rhomboidal airfoil $E_{r}=11 / 30$ tested in the A9 wind tunnel. Symbols indicate the pitch angles $\theta_{0}$ included in the analysis (see Fig. 25).

\subsection{Rhomboidal airfoil analysis}

The measurement test of the rhomboidal airfoil characteristics were performed in the A9 wind tunnel. The configuration of the body for the tests is shown in Fig. 23. Several rhomboidal airfoils of different relative thicknesses $E_{r}=10 / 30,11 / 30$ and $12 / 30$ were tested. The Reynolds number during the test, based on the chord of the airfoils $c=0.3 \mathrm{~m}$, was $\operatorname{Re}=1.03 \times 10^{5}$. The airfoil of relative thickness $E_{r}=11 / 30$ had rounded leading and trailing edges with a fillet radius of $r_{a}=0.52 \mathrm{~cm}$, which has an aerodynamic effect.

\subsubsection{Rhomboidal airfoil $E_{r}=11 / 30$}

Fig. 24 shows the $c_{l}(\alpha)$ and $c_{d}(\alpha)$ coefficients. As shown in Fig. 25 for the chosen dissipation $\tilde{p}_{d}$, in all the configurations there is only one solution, which is a stable one, and the range of the relative amplitude at equilibrium is $0.08<A^{*} / U^{*} \sim 0.11$ in the range of pitch angles $0.37<\theta_{0}<0.43 \mathrm{rad}$. 


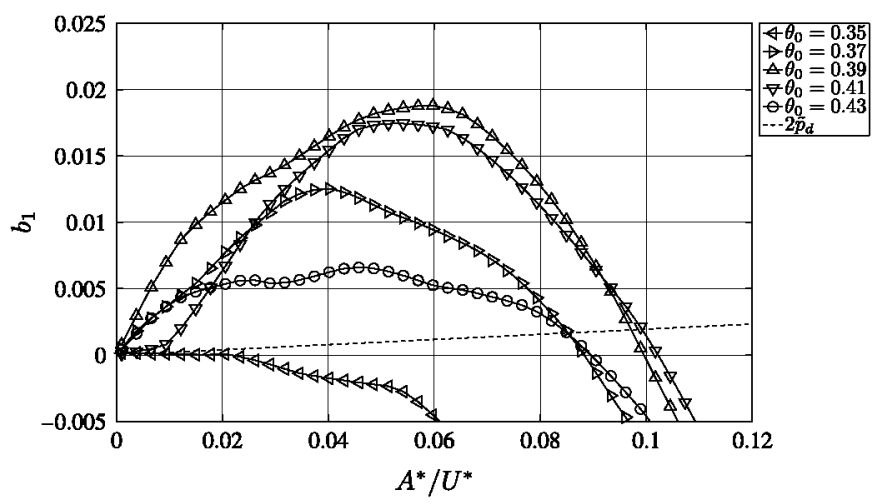

Fig. 25. Variation with the relative amplitude, $A^{*} / U^{*}$, of the coefficient $b_{1 n}$ obtained from the numerical integration, and the dissipation $\tilde{p}_{d}$, for the rhomboidal airfoil of $E_{\digamma}=11 / 30$ around $\theta=23^{\circ}=0.40$ rad tested in the $A 9$ wind tunnel.
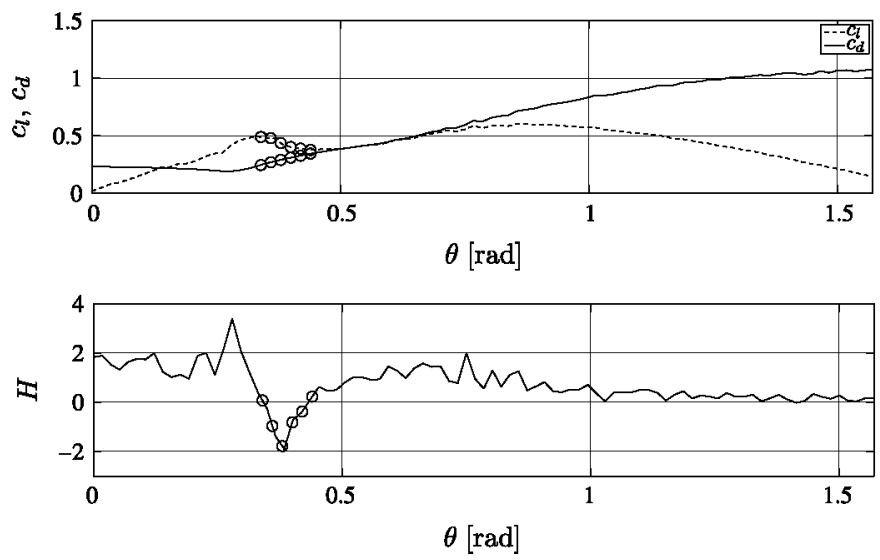

Fig. 26. Variation with the pitch angle of the body, $\theta$, of the coefficients of lift, $c_{l}$, and drag, $c_{d}$, and Glauert-Den Hartog parameter, $H$, over the rhomboidal airfoil $E_{r}=10 / 30$ tested in the $A 9$ wind tunnel. Symbols indicate the pitch angles $\theta_{0}$ included in the analysis (see Fig. 27).

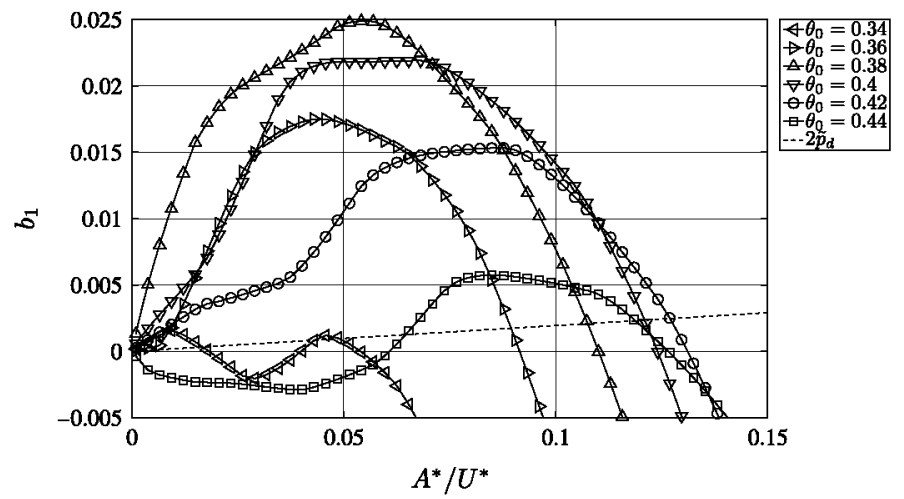

Fig. 27. Variation with the relative amplitude, $A^{*} / U^{*}$, of the coefficient $b_{1 n}$ obtained from the numerical integration, and the dissipation $\tilde{p}_{d}$, for the rhomboidal airfoil of $E_{r}=10 / 30$ around $\theta=23^{\circ}=0.40$ rad tested in the A9 wind tunnel.

\subsubsection{Rhomboidal airfoil $E_{r}=10 / 30$}

In this airfoil the intensity of the galloping is higher than for the relative thickness $E_{r}=11 / 30$, as can be appreciated in Fig. 26 ( $H$ is more negative than in Fig. 24) and the maximum value of $b_{1}$ is larger in Fig. 27 than in Fig. 25.

In Fig. 27, it can be seen that for the chosen dissipation, $\tilde{p}_{d}$, there is a stable solution in the range $0.34<\theta_{0}<0.44 \mathrm{rad}$, and also one unstable solution in the range $0.43<\theta_{0}<0.44 \mathrm{rad}$. At $\theta_{0}=0.34 \mathrm{rad}$ there are three solutions: one stable at $A^{*} / U^{*} \sim 0.02$ and a double solution at $A^{*} / U^{*} \sim 0.045$. At $\theta_{0}=0.44$ rad there are two solutions: one stable at $A^{*} / U^{*} \sim 0.12$ and the other one unstable at $A^{*} / U^{*} \sim 0.06$. 

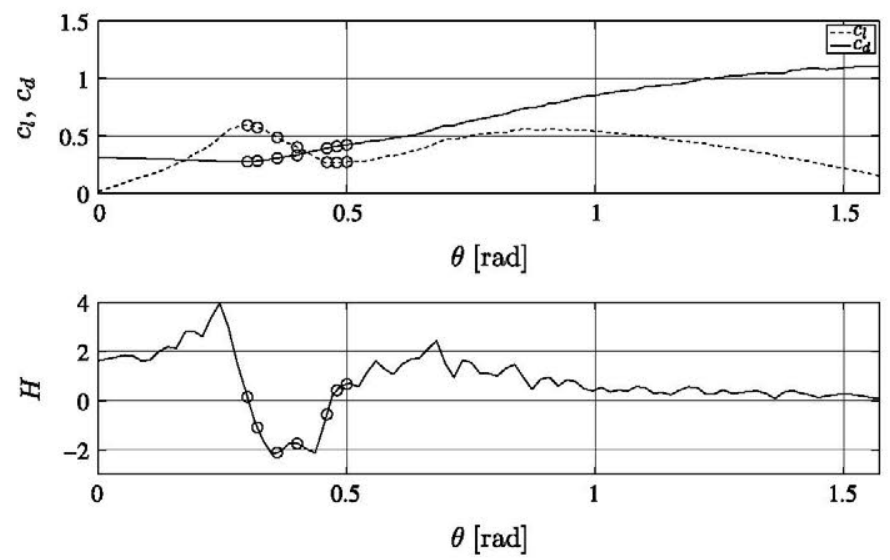

Fig. 28. Variation with the pitch angle of the body, $\theta$, of the coefficients of lift, $c_{l}$, and drag, $c_{d}$, and Glauert-Den Hartog parameter, $H$, over the rhomboidal airfoil $E_{r}=12 / 30$ tested in the $A 9$ wind tunnel. Symbols indicate the pitch angles $\theta_{0}$ included in the analysis (see Fig. 29).

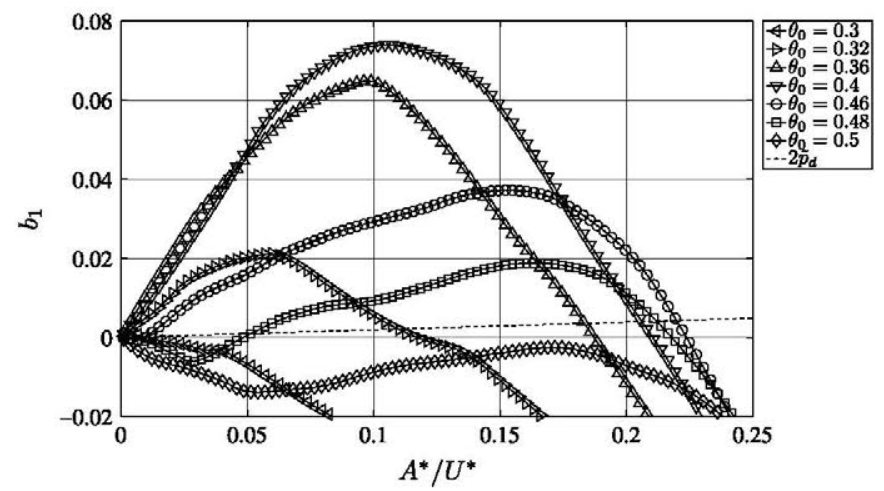

Fig. 29. Variation with the relative amplitude, $A^{*} / U^{*}$, of the coefficient $b_{1 n}$ obtained from the numerical integration, and the dissipation $\tilde{p}_{d}$, for the rhomboidal airfoil of $E_{r}=12 / 30$ around $\theta=23^{\circ}=0.40 \mathrm{rad}$ tested in the A9 wind tunnel.

\subsubsection{Rhomboidal airfoil $E_{r}=12 / 30$}

In this airfoil, the range of pitch angles of galloping is wider than for the other of smaller relative thickness, as can be seen in Fig. 28. In Fig. 29, it can be observed that for the chosen dissipation, $\tilde{p}_{d}$, there is one stable solution in the range $0.32<\theta_{0}<0.48 \mathrm{rad}$, the maximum of the relative amplitude of equilibrium $A^{*} / U^{*}$ appears at $\theta_{0}=0.46 \mathrm{rad}$. There is also an unstable solution in the range $0.46<\theta_{0}<0.48 \mathrm{rad}$.

\subsection{Triangles}

In Fig. 30 the dimensionless extracted power, for the bodies studied, are presented. In the same figure the dimensionless power curves calculated from the results reported in Alonso et al. (2012) for triangular cross-section bodies are included. It can be seen that the rhomboidal cross-section of relative thickness $E_{r}=11 / 30$ has the minimum capacity of energy extraction from the flow, and this capacity, as in the case with the biconvex airfoil, is negligible compared with the extracted energy of the D-shape and the triangle cross-section bodies. The triangle of $\beta=60^{\circ}$, where $\beta$ is the angle of the isosceles triangle (equilateral in this case) seems to be the most efficient cross-section shape.

It is worth noting the difference between the results obtained for the maximum specific extracted power, $\tilde{p}_{\text {emax }}$ (see Fig. 31), and the maximum dimensionless extracted power, $p_{\text {emax }}$. The triangle of $\beta=60^{\circ}$ can extract the highest amount of energy from the fluid flow, and the D-shape cross-section body provides the highest specific extracted power, $\tilde{p}_{e}$. This is so because the relative amplitude $A^{*} / U^{*}$ needed to generate such power is smaller than in the case of the triangular crosssection body. This information can be helpful in the design process of a device extracting energy by transverse galloping.

\subsection{Square}

The method has also been used to compare the suitability of the 7th and higher order polynomials to fit the experimental data reported by $\mathrm{Ng}$ et al. (2005) for square-section cylinders, see Fig. 32 . The results obtained by numerical integration $b_{1 n 7}$ of the 7th order polynomial proposed by Parkinson and Smith (1964) and the integration of the experimental results $b_{1 n e}$ are 


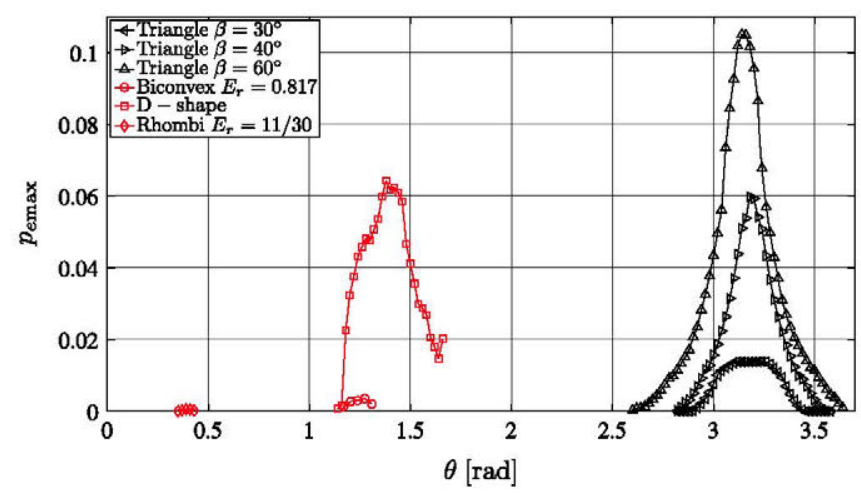

Fig. 30. Variation with the pitch angle, $\theta$, of the maximum dimensionless extracted power, $p_{\text {emax }}$, for different cross-section bodies.

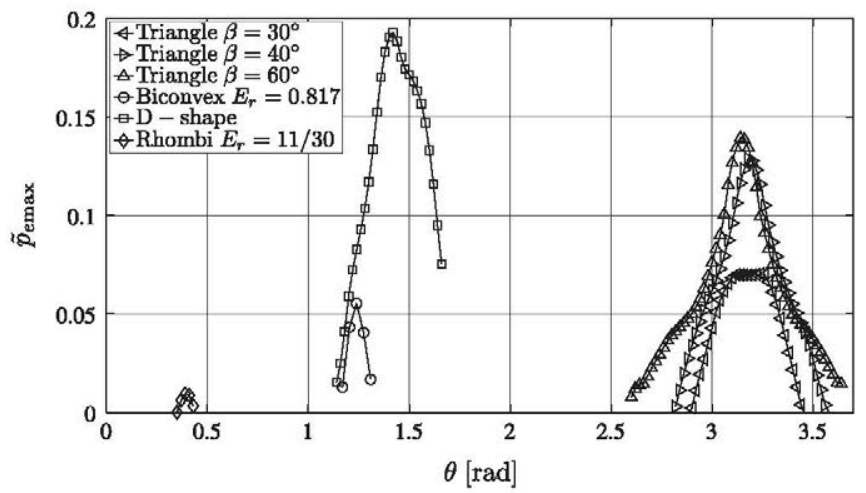

Fig. 31. Variation with the pitch angle, $\theta$, of the maximum specific extracted power, $\tilde{p}_{\text {emax }}$, for different cross-section bodies.

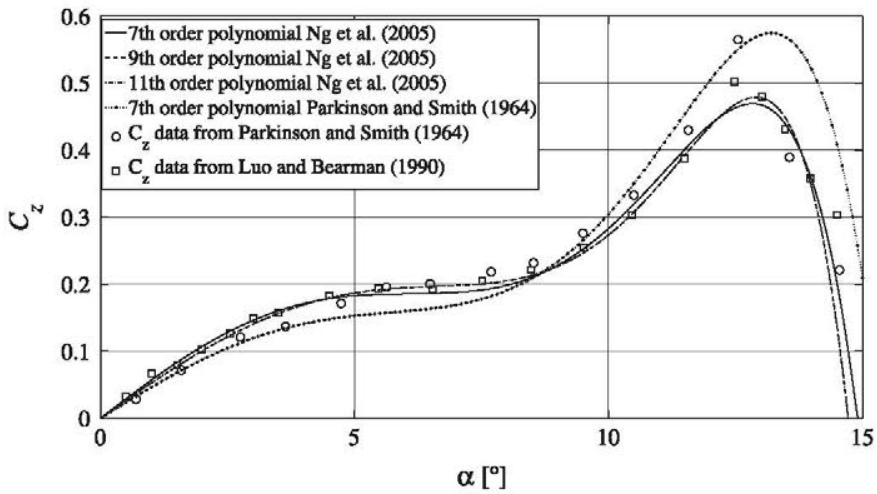

Fig. 32. Variation of the transverse force coefficient, $C_{z}$, with the angle of attack, $\alpha$, experimental data from Ng et al. (2005), Parkinson and Smith (1964), Luo and Bearman (1990) and Parkinson and Brooks (1961). In this figure, the 9th and 11th order polynomials of Ng et al. (2005) are coincident.

presented in Fig. 33. Around a 5\% difference can be appreciated in the maximum value of $b_{1}$, due to the fact that the fitting of this polynomial is not accurate enough, compare dotted line and circular symbols in Fig. 32. The difference in the position of the inflection point is significant. The position of inflection point is an important characteristic, regarding the dynamical behaviour.

In Fig. 34 a better polynomial fitting of the experimental data (given by Luo and Bearman, 1990) is observed, and the difference between the integration of the experimental data and the polynomials is small in the range of relative amplitude $A^{*} / U^{*}$, from 0 to 0.25 . However, at higher relative amplitudes differences between the results from 7 th and 9 th order polynomial fittings can be appreciated. It is also shown that 9 th and 11 th order polynomials give practically the same results.

In order to compare the $b_{1}$ curves for higher amplitudes, the lift $c_{l}$ and drag $c_{d}$ coefficient data for a square cylinder have been obtained from Parkinson and Brooks (1961) because there are defined till angle of attack $\alpha=20^{\circ}=0.3491$ rad. The experimental values of $C_{z}(\alpha)$ are obtained from $c_{l}$ and $c_{d}$ data using Eq. (2), and have been fitted by 7 th, 9th and 11th order 


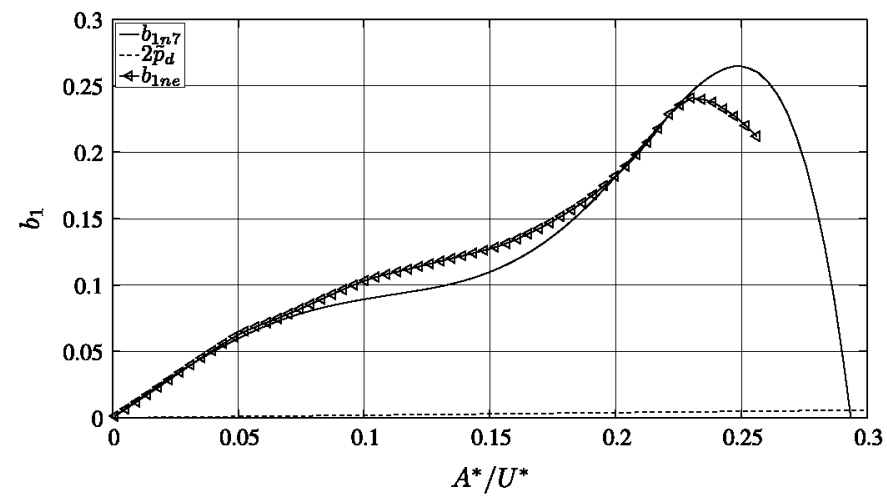

Fig. 33. Variation with the relative amplitude, $A^{*} / U^{*}$, of the coefficient $b_{1}$ obtained by direct numerical integration of experimental data, $b_{1 n e}$; by integration of a 7 th order polynomial, $b_{1 n 7}$; and the specific dissipated power, $\tilde{p}_{d}$, for a square-cylinder. Polynomial coefficients $\left(a_{1}=2.69, a_{3}=-168\right.$, $a_{5}=6270, a_{7}=59900$ at $\operatorname{Re} \sim 22300$ ) from Parkinson and Smith (1964).

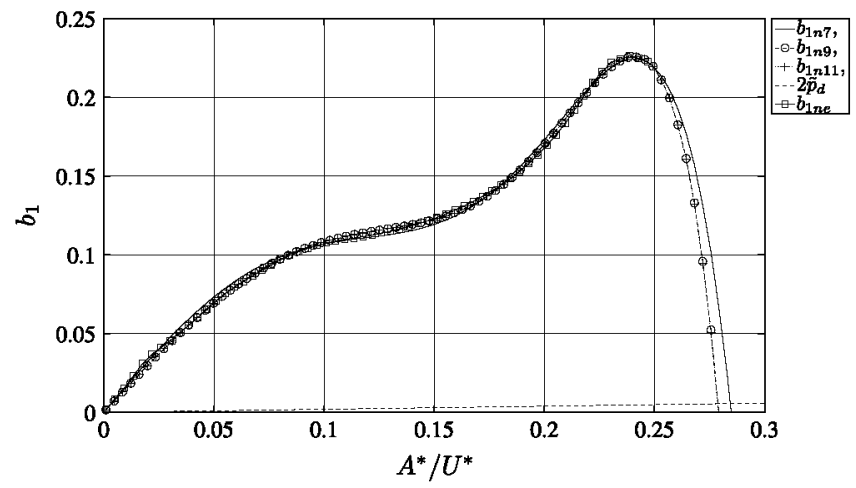

Fig. 34. Variation with the relative amplitude, $A^{*} / U^{*}$, of the coefficient $b_{1}$ obtained by direct numerical integration of 7 th, 9 th and 11 th order polynomials, $b_{1 n 7}, b_{1 n 9}$ and $b_{1 n 11}$, respectively; experimental data, $b_{1 n e}$; and the specific dissipated power, $\tilde{p}_{d}$, for a square-cylinder. Experimental data and polynomial coefficients extracted from $\mathrm{Ng}$ et al. (2005).

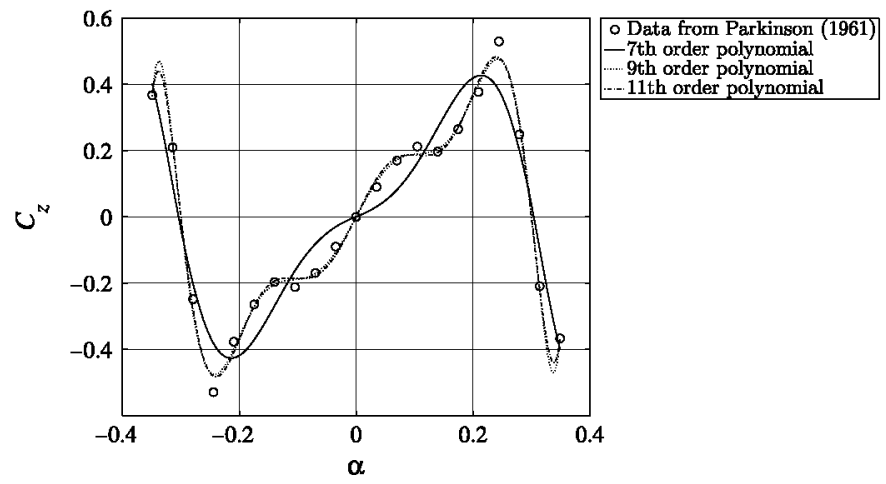

Fig. 35. Variation of the transverse force coefficient $C_{z}$, with the angle of attack $\alpha$ (from Parkinson and Brooks, 1961) and 7 th, 9th and 11th order polynomials that fit the experimental data, obtained with a Matlab function (polyfit), see Table 3.

polynomials. To do this, a Matlab function has been employed. The results are shown in Fig. 35 . For convenience, the coefficients are given in reference to the maximum relative amplitude $\left(\tan ^{-1}\left(A^{*} /\left.U^{*}\right|_{\max }\right)=\alpha_{m}=0.3491\right)$ as follows:

$$
C_{z}(\alpha)=\sum a_{n} \alpha_{m}^{n}\left(\frac{\alpha}{\alpha_{m}}\right)^{n}=\sum a_{n}^{\prime}\left(\frac{\alpha}{\alpha_{m}}\right)^{n}
$$

and the results are listed in Table 3.

The 7th order polynomial shows an "out of phase" behaviour, as the curvature has variations opposite to the trends of the experimental curve. The behaviour is also shown by comparing the signs of coefficients $a_{i}^{\prime}$ in Table 3 . The 9th and 11 th order 
Table 3

Coefficients of 7 th, 9th, 11 th order polynomials obtained with Matlab function polyfit for the fitting of the experimental data $C_{z}(\alpha)$ obtained from Parkinson and Brooks (1961).

\begin{tabular}{lrrrrc}
\hline \multicolumn{1}{l}{$a_{1}^{\prime}$} & \multicolumn{1}{c}{$a_{3}^{\prime}$} & \multicolumn{1}{c}{$a_{5}^{\prime}$} & \multicolumn{1}{c}{$a_{7}^{\prime}$} & $a_{9}^{\prime}$ & \multicolumn{1}{c}{$a_{11}^{\prime}$} \\
\hline 0.27 & 3.79 & -8.63 & 4.17 & 0 & 0 \\
1.15 & -8.72 & 38.61 & -60.90 & 29.49 & 0 \\
1.25 & -11.01 & 52.89 & -95.62 & 65.98 & -13.79 \\
\hline
\end{tabular}

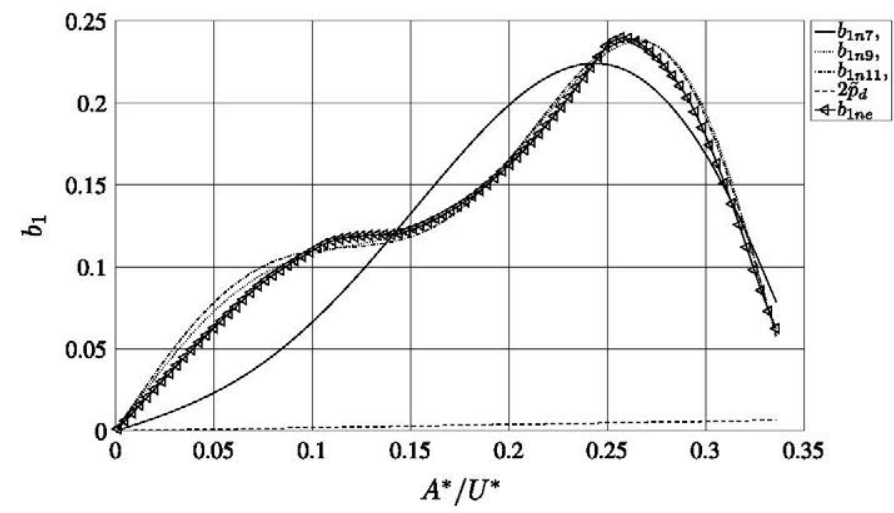

Fig. 36. Variation with the relative amplitude, $A^{*} / U^{*}$, of the coefficient $b_{1}$ obtained by numerical integration of 7 th, 9 th and 11 th order polynomials, $b_{1 n 7}$, $b_{1 n 9}$ and $b_{1 n 11}$, respectively; experimental data, $b_{1 n e}$; and the specific dissipated power, $\tilde{p}_{d}$, for a square-cylinder. The lift $c_{l}$ and drag $c_{d}$ coefficients to obtain $C_{z}$ were taken from Parkinson and Brooks $(1961)$, and $C_{z}(\alpha)$ is shown in Fig. 35.

polynomials fit remarkably well the trends of the experimental points, except the last points, where some overshoot is present.

In Fig. 36 a good agreement between $b_{1}$ obtained from integration of both 9th and 11 th order polynomials, $b_{1 n 9}$ and $b_{1 n 11}$ respectively, and the integration of the experimental data, $b_{1 \text { ne }}$, can be shown. The integration of the 7th order polynomial, $b_{1 n 7}$, does not show a good agreement in this case (see also Fig. 36).

Concerning the square cylinder case, some conclusions can be drawn. Although the results from polynomial fitting and direct numerical integration from experimental data, $b_{1 n 7}$ and $b_{1 \text { ne }}$, respectively, shown in Fig. 33 do not coincide, the qualitative variation seems to be in agreement. Therefore the analysis of the stability presented in Section 4 would not be compromised, at least qualitatively.

However, if the amplitude range is increased (see results in Figs. 35 and 36), the 7th order polynomial gives clearly trends and values different from both the experimental results $b_{1 n e}$ and polynomial fittings $b_{1 n a}$ and $b_{1 n 11}$. Furthermore, both $b_{1 n 9}$ and $b_{1 n 11}$ results start to fail close to the ends of the amplitude range (see Fig. 35) while the fitting was quite satisfactory in Fig. 32, for smaller amplitude range.

Therefore, it can be deduced that no rule can be given for the order of polynomial to be used in a general case, taking into account the amplitude range, and the suitability should be assessed by qualitative feeling, in a case-by-case basis.

This inconvenience is not shown by the method based on the direct numerical integration of the experimental results, presented in this paper, as an intermediate polynomial fitting procedure is not needed.

\section{Conclusions}

In this paper a new method to analyse the stability of transverse galloping based on stationary measurements performed in a wind tunnel has been presented. This method does not make use of a polynomial fitting of the experimental data. The influence of the discretization interval of the measurements in the uncertainty of equilibrium positions has been analysed and quantified. The numerical method presented has been used to analyse the stability of cylindrical bodies with different cross sections (biconvex, rhomboid, D-shape, triangles and squares), and their respective extracting power capabilities have been compared.

By analysing the extracted power curves of a body in the neighbourhood of the points prone to present galloping (which are only dependent on the aerodynamic characteristic of the body), the value of the relative oscillation amplitude $A^{*} / U^{*}$ that gives rise to the maximum extracted power can be determined, and as a consequence the associated mechanical parameter $m^{*} \zeta / U^{*}$ can be deduced. As it is a combination of the values of wind speed, spring stiffness and damping coefficient, the appropriate values of these variables required to maximize energy extraction from the flow can be determined. In this way, the design process is decomposed into two independent phases and thus is simplified considerably. 
It has been found that same differences appear in both the maximum extracted power and in the relative amplitude at equilibrium, between the results of the direct integration of experimental data and those of the integration of the polynomial fitted to the same experimental data. These differences appear because the polynomial fitting is an approximation to the real curve $C_{z}(\alpha)$ and thus, if the polynomial fitting is no good enough, differences come out.

In this regard, the new method avoids problems related to polynomial fitting, for instance the polynomial order suitable for a given application. Besides, for the determination of maximum extracted power, the associated analytical treatment using a high order polynomial is not practical. Furthermore, if the polynomial fitting is not enough accurate the prediction of the dynamical behaviour of the analysed bodies can be wrong.

The authors wish to thank Prof. J. Meseguer and G. Alonso for their helpful comments.

Abdelkefi, A., Yan, Z., Hajj, M.H., 2013. Modeling and nonlinear analysis of piezoelectric energy harvesting from transverse galloping. Smart Materials and Structures 22, 025016.

Alonso, G., Sanz-Lobera, A., Meseguer, J., 2012. Hysteresis phenomena in transverse galloping of triangular cross-section bodies. Journal of Fluids and Structures 33, 243-251.

Alonso, G., Valero, E., Meseguer, J., 2009. An analysis on the dependence on cross section geometry of galloping stability of two-dimensional bodies having either biconvex or rhomboidal cross sections. European Journal of Mechanics-B/Fluids 28, 328-334.

Barrero-Gil, A., 2008. Dinámica de osciladores aeroelásticos. Ph.D. Thesis. Escuela Técnica Superior de Ingenieros Aeronáuticos/Universidad Politécnica de Madrid.

Barrero-Gil, A., Sanz-Andres, A., Alonso, G., 2009. Hysteresis in transverse galloping: the role of the inflection points. Journal of Fluids and Structures 25 , 1007-1020.

Crawley, F.E., Curtiss Jr, H.C., Peters, D.A., Scanlan, R.H., Sisto, F., 1995. A Modern Course in Aeroelasticity. Kluwer Academic Publishers.

Luo, S., Bearman, P., 1990. Predictions of fluctuating lift on a transversely oscillating square-section cylinder. Journal of Fluids and Structures 4, 219-228.

Ng, Y., Luo, S., Chew, Y., 2005. On using high-order polynomial curve fits in the quasi-steady theory for square-cylinder galloping. Journal of Fluids and Structures 20, 141-146.

Parkinson, G.V., Brooks, N.P.H., 1961. On the aeroelastic instability of bluff cylinders. Journal of Applied Mechanics, 252-258.

Parkinson, G.V., Smith, J.D., 1964. The square prism as an aeroelastic non-linear oscillator. Quarterly Journal of Mechanics and Applied Mathematics 17 , 225-239. 